\title{
VERTEBRATE FAUNA IN CAVES OF EASTERN TENNESSEE WITHIN THE APPALACHIANS KARST REGION, USA
}

\author{
Matthew L. Niemiller ${ }^{*}$, Kirk S. Zigler², Charles D.R. Stephen³, Evin T. Carter4, \\ Audrey T. Paterson 5 , Steven J. Taylor ${ }^{1}$, and Annette Summers Engel 5
}

\begin{abstract}
More than one-fifth of the documented caves in the United States occur in Tennessee. The obligate subterranean biota of Tennessee is rich and diverse, with 200 troglobionts reported from over 660 caves. Fifty troglobionts are known from just 75 of the 1,469 caves in the Appalachian Valley and Ridge physiographic province of eastern Tennessee. Tennessee's Valley and Ridge has been under-sampled relative to other karst areas in the state, limiting our knowledge of cave and karst species diversity and distributions and compromising our ability to identify habitats and species potentially at risk from anthropogenic threats, such as urban sprawl near the metropolitan area of Knoxville. Knowledge of nontroglobiontic species inhabiting caves, including vertebrates, is particularly sparse in this region. Although caves have long been recognized as critical habitats for several bat species, the importance of caves for other vertebrate taxa has received less attention. Caves are important habitats for many other nontroglobiontic vertebrates and should be considered in the management and conservation of these species. Our decade-long study bioinventoried 56 caves in 15 counties and begins to address knowledge gaps in distributions and cave use by vertebrates in the Valley and Ridge and adjacent Blue Ridge Mountains of eastern Tennessee within the Appalachians karst region. In addition, we conducted a thorough review of the literature and museum databases for additional species-occurrence records in those provinces of eastern Tennessee. From these sources, we present an annotated list of 54 vertebrate taxa, including 8 fishes, 19 amphibians ( 8 anurans and 11 salamanders), 6 reptiles, 3 birds, and 18 mammals. Three species are included on the IUCN Red List of Threatened Species, while six species are at risk of extinction based on NatureServe conservation rank criteria. Ten bat species are known from 109 caves in 24 eastern Tennessee counties. Our bioinventories documented five bat species in 39 caves, including new records of the federally endangered Gray Bat (Myotis grisescens). We observed visible evidence of whitenose syndrome caused by the fungal pathogen Pseudogymnoascus destructans at four caves in Blount, Roane, and Union counties. We documented two new localities of the only troglobiontic vertebrate in the Valley and Ridge, the Berry Cave Salamander (Gyrinophilus gulolineatus). Despite these efforts, significant sampling gaps remain —only $7.7 \%$ of known caves in the Valley and Ridge and Blue Ridge Mountains of eastern Tennessee have records of vertebrate-species occurrence. Moreover, few caves in eastern Tennessee have experienced repeated, comprehensive bioinventories, with the exception of periodic surveys of hibernating bats at selected caves. Future bioinventory efforts should incorporate multiple visits to individual caves, if possible, and more efforts should focus on these understudied areas of eastern Tennessee.
\end{abstract}

\section{INTRODUCTION}

Caves and karst habitats are important in varying degrees to many vertebrate species, most of which are not troglobionts. Many species of amphibians and mammals use caves for foraging, reproduction, or refuges from harsh conditions on the surface during periods of drought or extreme temperatures. Several species of bats, such as Myotis grisescens, roost in caves during the summer in large colonies or use caves for hibernation during the winter. Caves are the sites of reproduction and nesting for several species of salamanders. Some fish and salamander species that occur in surface habitats, such as Cottus carolinae and Gyrinophilus porphyriticus, can also live their entire lives in caves. Understanding the distribution and extent of cave utilization is important for proper conservation and management of these vertebrate species, but few

\footnotetext{
*Corresponding author: cavemander17@gmail.com

${ }^{1}$ Illinois Natural History Survey, Prairie Research Institute, University of Illinois Urbana-Champaign, Champaign, IL 61820

${ }^{2}$ Department of Biology, The University of the South, Sewanee, TN 37383

${ }^{3}$ Department of Biological Sciences, Auburn University, Auburn, AL 36849

${ }^{4}$ Department of Ecology and Evolutionary Biology, University of Tennessee, Knoxville, TN 37996

${ }^{5}$ Department of Earth and Planetary Sciences, University of Tennessee, Knoxville, TN 37996
} 
comprehensive, annotated lists of extant vertebrate cave fauna have been published for the eastern United States. Cliburn and Middleton (1983) discussed the vertebrate fauna of Mississippi caves, and Garton et al. (1993) reviewed the vertebrate cave fauna of West Virginia. Faunal lists at varying geographic scales have been published for taxa such as fishes (Poly, 2001; Poly and Boucher, 1996) and amphibians and reptiles (Himes et al., 2004; Niemiller and Miller, 2009).

More caves are known in Tennessee than in any other state in the United States, with over 10,000 documented caves as of 2015 occurring in two major karst regions, the Interior Low Plateau and the Appalachians (Niemiller and Zigler, 2013). The Appalachians karst region extends from southeastern New York to northwestern Georgia and northeastern Alabama. In Tennessee, the Appalachians karst region includes karst within the Valley and Ridge province and Blue Ridge Mountains. The Valley and Ridge is flanked to the west by the Cumberland Plateau and to the east by the Blue Ridge Mountains, which contain limited exposures of karst. The Appalachians karst region is the second largest karst area in Tennessee, after the Highland Rim of the Interior Low Plateau.

Bioinventories of the subterranean fauna of Tennessee have been conducted for more than a century (e.g., Cope and Packard, 1881; Hay, 1902; Lewis, 2004, 2005; Lewis et al., 2010; Dixon and Zigler, 2011; Wakefield and Zigler, 2012). Most records are included in a report by Holsinger and Culver (1988) summarizing invertebrate cave fauna from Valley and Ridge bioinventories conducted between 1961 and 1980 in seven northeastern Tennessee counties and from approximately five hundred caves in Virginia. In the Blue Ridge Mountains of eastern Tennessee, studies of cave fauna are limited to those in and immediately adjacent to Great Smoky Mountains National Park (Wallace, 1984, 2003; Reeves, 2000; Dodd et al., 2001; Mays, 2002; Taylor and Mays, 2006). Niemiller and Zigler (2013) recently compiled over twenty-two hundred records of obligate subterranean species in Tennessee, and listed 200 invertebrate and vertebrate troglobiontic species identified from 661 caves in the state. As of 2013, troglobiontic faunal records exist for just 5.1\% of known caves (75 of $1,469)$ in the Valley and Ridge province of eastern Tennessee and $7.6 \%$ of known caves (13 of 171) in the Blue Ridge Mountains (Niemiller and Zigler, 2013). From these karst regions, only 50 and 9 troglobiontic species have been documented, respectively.

Vertebrate cave fauna records of the Appalachians karst region in eastern Tennessee are largely limited to distributional records of cave-roosting bat species documented in state biological databases, such as those of Tennessee Natural Heritage Inventory Program and other government reports, and to studies of salamanders, mainly from caves in Great Smoky Mountains National Park (Dodd et al., 2001; Taylor and Mays, 2006) and particularly the Berry Cave Salamander Gyrinophilus gulolineatus (e.g., Miller and Niemiller, 2007,
2008; Niemiller et al., 2010). Considerable cave biodiversity remains to be discovered and documented. The Appalachians karst region has been under-sampled compared to many other regions of the state (Niemiller and Zigler, 2013). To begin filling in sampling gaps in Valley and Ridge and adjacent karst in the Blue Ridge Mountains of eastern Tennessee, we inventoried the fauna of 56 caves in 15 counties between October 23, 2004 and August 3, 2015, with the intention of creating annotated lists of vertebrates and invertebrates that will supplement faunal surveys that have already been published or that might be underway. The invertebrate fauna of the Appalachians karst region of eastern Tennessee will be addressed separately. For the vertebrate fauna lists, we compiled cave occurrence records for these areas of eastern Tennessee from a variety of published and unpublished literature sources, databases, and museum collections. We include these data to produce the first comprehensive faunal list of vertebrates from caves in this region.

\section{Materials ANd Methods}

We conducted bioinventories of 56 caves representing 107 cave visits within the Appalachian Valley and Ridge and adjacent Blue Ridge Mountains of eastern Tennessee (Table 1, Fig. 1). Many of these caves had never been bioinventoried. Survey sites included Blount (1 cave), Campbell (1), Carter (2), Claiborne (3), Grainger (1), Jefferson (2), Knox (21), Loudon (3), McMinn (2), Meigs (1), Monroe (6), Rhea (1), Roane (5), Sevier (1), and Union (6) counties. Cave descriptions, maps, and locations are maintained by the Tennessee Cave Survey (http://www. subworks.com/tcs/), and we report the TCS cave inventory number with the cave name. We emphasized Knox County, where Gyrinophilus gulolineatus may potentially exist and could be at risk from anthropogenic impacts.

Bioinventories involved visual-encounter surveys for cave life in terrestrial, riparian, and aquatic habitats, such as entrance areas, cave walls and ceilings, mud banks, rimstone pools, and cave streams. These surveys systematically traversed the cave from the entrance to the farthest extent of the cave explorable by the research team. Search effort included lifting rocks and other cover, as well as searching through cobbles, detritus, and organic matter. For fishes, amphibians, and reptiles, we made a concerted effort to capture each individual observed either by hand or with dip nets to confirm identification and obtain voucher photographs. Depending on the extent of the cave system, each survey typically involved 2 to 4 surveyors (maximum 12), with a search effort of 2 to 36 person-hours per cave visit.

All statistical analyses were conducted in the $\mathrm{R}$ statistical computing environment (v.3.1.2; R Core Team, 2014). We explored possible relationships between species richness and horizontal length of caves, a proxy for available habitat because the caves sampled were predominantly horizontal in development, and between species richness and the number of bioinventories conducted at a cave using 
Pearson correlation analyses. A weighted Pearson correlation analysis in the $\mathrm{R}$ package weights (v.0.80; Pasek, 2015) was employed to explore the relationship between species richness and cave length weighted by the number of bioinventories conducted at each cave. We also employed a Wilcoxon rank sum test to assess if wet caves differed from dry caves with respect to species diversity. Caves bioinventoried were classified as either wet or dry, where each cave was classified based on the dominant habitat type present, aquatic or terrestrial. Of the 56 caves bioinventoried, 36 caves were classified as wet and 20 caves as dry. When applicable, we report mean values \pm standard deviation.

We searched for additional distributional records of vertebrates in eastern Tennessee caves in the scientific literature, unpublished reports, biodiversity databases, and museum accession records. Literature sources included peer-reviewed journals, books, proceedings, theses and dissertations, government reports, and caving organization newsletters. Searches of literature sources included keyword queries of ISI Web of Science, Google Scholar, and Zoological Record. Database sources included biodiversity databases maintained by the Tennessee Natural Heritage Inventory Program (TNNH) and the Bat Population Database. We also queried the VertNet database (http:// www.vertnet.org), a web portal to search accessions of over 170 vertebrate museum collections from 12 countries. Institutions for which accessions included specimens collected from eastern Tennessee include Carnegie Museum of Natural History (CM), Kansas University Biodiversity Institute (KU), Museum of Vertebrate Zoology at University of California-Berkeley (MVZ), North Carolina Museum of Natural Sciences (NCSM), Sam Noble Oklahoma Museum of Natural History (OMNH), Santa Barbara Museum of Natural History (SBMNH), Museum of Texas Tech University (TTU), University of Michigan Museum of Zoology (UMMZ), Smithsonian Institution National Museum of Natural History (USNM), and Western Foundation of Vertebrate Zoology (WFVZ). We attempted to georeference each distributional record using the TCS database of caves.

The annotated list of vertebrate fauna includes the scientific name, authority, ecological classification, common name, and conservation status for each species. Taxonomic nomenclature primarily followed the Encyclopedia of Life (http://www.eol.org). We used common names from published sources when available (e.g., Etnier and Starnes, 1993; Niemiller and Reynolds, 2011; Niemiller et al., 2013). Classifications of cave-associated organisms (cavernicoles) have been proposed by several authors (e.g., Barr, 1968; Sket, 2008; Culver and Pipan, 2009). We used terminology from Barr (1968) with clarification from Sket (2008) and Culver and Pipan (2009), depending on the taxa, to indicate species found in terrestrial (troglo-) versus aquatic (stygo-) habitats. The four primary ecological categories, with the abbreviations used in the fauna list below and Table 2, were troglobiont (synonym: troglobite) or stygobiont (synonym: stygobite) (TB or SB, respectively), troglophile or stygophile (TP or SP) (synonym: eutroglophile), trogloxene or stygoxene (TX or SX) (synonym: subtroglophile), and accidental (AC) (synonym: trogloxene, sensu Sket, 2008). Troglobionts and stygobionts are obligate cavernicoles with morphological, physiological, and behavioral adaptations for living in subterranean habitats and that have few to no records from surface habitats. Troglophiles and stygophiles frequent subterranean habitats and are capable of completing their life cycles within caves but also may occur in surface habitats. Trogloxenes and stygoxenes use subterranean habitats seasonally, or for only a portion of their life cycles, but also rely significantly on surface habitats. Accidentals are species found in caves only by accident, such as by falling into a pit or being washed into a cave during a flood.

The conservation status of each species, based on the International Union for Conservation of Nature (IUCN) Red List of Threatened Species (http://www.iucnredlist.org/ [accessed June 29, 2015]) and NatureServe (http://www. natureserve.org/ [accessed June 29, 2015]), is included to provide a better understanding of the distribution and biogeography of subterranean organisms in eastern Tennessee, and to aid in the future conservation and management of this unique fauna. The status of a species according to the U.S. list of threatened and endangered species under the Endangered Species Act is included (http://www. fws.gov/endangered), as well as if a species is included on the list of rare animals in Tennessee (Withers, 2009). Seven IUCN Red List categories are recognized on a continuum of increasing extinction risk (International Union for the Conservation of Nature, 2012): Least Concern, Near Threatened, Vulnerable, Endangered, Critically Endangered, Extinct in the Wild, and Extinct. Two additional categories are also recognized: Data Deficient, in which a species has been evaluated, but insufficient data are available to make a determination on conservation rank; and Not Evaluated, in which a species has yet to be evaluated. Critically Endangered, Endangered, and Vulnerable are considered Threatened categories. Species are classified as Threatened provided they meet one of five criteria (International Union for the Conservation of Nature, 2012): (A) past, present, or projected reduction in population size over three generations; (B) small geographic range in combination with fragmentation, population decline or fluctuations; (C) small population size in combination with decline or fluctuations; (D) very small population or very restricted distribution; and (E) a quantitative analysis of extinction risk. The IUCN Red List classification and associated criteria and subcriteria are presented, if applicable. Subcriteria are detailed in International Union for the Conservation of Nature (2012). NatureServe conservation status ranks are based on a one to five scale, from most to least at risk of extinction (Faber-Langendoen et al., 2012): G1 (Critically Imperiled), G2 (Imperiled), G3 (Vulnerable), G4 (Apparently Secure), and G5 (Secure). 
Table 1. List of caves bioinventoried in the Appalachian Valley and Ridge and adjacent Blue Ridge Mountains of eastern Tennessee, including survey dates, Tennessee Cave Survey (TCS) cave number, and number of species documented for five classes of vertebrates observed: $\mathbf{F}$ - fishes; A - amphibians; $\mathbf{R}$ - reptiles; B - birds; and mammals (separated into bats and NM, non-bat mammals).

\begin{tabular}{|c|c|c|c|c|c|c|c|c|c|c|}
\hline County & Cave & TCS No. & Date & $\mathrm{F}$ & A & $\mathrm{R}$ & $\mathrm{B}$ & NM & Bats & Total \\
\hline Blount & Tuckaleechee Caverns & BA11 & 2014: 20 Mar & & 1 & & & & 2 & 3 \\
\hline Campbell & Panther Cave No. 1 & CM8 & 2015: $23 \mathrm{Mar}$ & & 4 & & 1 & 2 & 2 & 9 \\
\hline Carter & Carter Saltpeter Cave & CR1 & 2014: 14 Мay & & 1 & 1 & & & 1 & 3 \\
\hline Carter & Rockhouse Cave & CR3 & 2014: 14 May & & & & & & & 0 \\
\hline Claiborne & Sour Kraut Cave & CB46 & 2015: 1 Jun & & 1 & & 1 & 1 & & 3 \\
\hline Claiborne & Buis Saltpeter Cave & CB48 & 2015: 1 Jun & & 1 & & 1 & 1 & & 3 \\
\hline Claiborne & Kings Saltpeter Cave & CB52 & 2015: 30 May & & 2 & & 1 & 1 & & 4 \\
\hline Grainger & Indian Cave & GA4 & 2014: 22 Feb; 29 Jun & 1 & 3 & & & & 3 & 7 \\
\hline Jefferson & Tater Cave & JF8 & 2015: 3 Aug & & 2 & & & 1 & 1 & 4 \\
\hline Jefferson & Silo Pit Cave & JF71 & 2015: 3 Aug & & 3 & 1 & & & & 4 \\
\hline Knox & Campbell Cave & KN1 & 2014: $23 \mathrm{Dec}$ & & & & 1 & 2 & 2 & 5 \\
\hline Knox & Cherokee Bluff Cave & KN4 & 2015: 7 Mar & & & & & & 1 & 1 \\
\hline Knox & Mudflats Cave & KN9 & $\begin{array}{l}\text { 2004: } 20 \text { Nov } \\
\text { 2005: } 6 \text { Jan; } 30 \text { Dec } \\
\text { 2006: } 12 \text { Nov } \\
\text { 2007: } 7 \text { Jun } \\
\text { 2008: } 2 \text { Oct } \\
\text { 2014: } 5 \text { Apr: } 20 \text { Oct }\end{array}$ & 1 & 4 & & & 1 & 2 & 8 \\
\hline Knox & Carter Cave & KN14 & 2008: 21 May & & 3 & & & 1 & 1 & 5 \\
\hline Knox & Keller Bend Cave & KN16 & 2013: 16 May & & 1 & & & 1 & 1 & 3 \\
\hline Knox & Blowing Hole Cave & KN19 & 2013: 16 May & & 3 & & & 1 & 1 & 5 \\
\hline Knox & Cherokee Caverns & KN22 & 2014: 5 Apr & & 1 & & & & 1 & 2 \\
\hline Knox & Cruze Cave & KN24 & $\begin{array}{l}\text { 2004: } 31 \text { Oct } \\
\text { 2005: } 6 \text { Jan; } 6 \text { Mar; } 31 \text { Dec } \\
\text { 2006: } 18 \text { Jul; } 10 \text { Sep; } 19 \text { Nov } \\
\text { 2008: } 19 \text { May; } 7 \text { Jul } \\
\text { 2013: } 13 \text { May; } 15 \text { Jun } \\
\text { 2014: } 10 \text { Apr; } 11 \text { May; } 19 \text { Jun; } \\
\text { 14 Aug; } 13 \text { Oct }\end{array}$ & & 4 & & & 1 & 2 & 7 \\
\hline Knox & Meads Quarry Cave & KN28 & $\begin{array}{l}\text { 2004: } 23 \text { Oct } \\
\text { 2006: } 4 \text { Nov } \\
\text { 2007: } 22 \text { Apr; } 9 \text { Sep; } 8,24 \text { Nov } \\
\text { 2008: } 24 \text {, } 31 \text { Jan; 1, 6, } 30 \text { Mar; } \\
\text { 10, } 30 \text { Apr; 15 May; 4, } 27 \text { Jun; } \\
\text { 30 Jul; } 10 \text { Sep } \\
\text { 2013: } 5 \text { Oct }\end{array}$ & & 8 & 1 & & 2 & 3 & 14 \\
\hline Knox & Christian Cave & KN49 & 2005: 17 Sep & 2 & 3 & & & & 2 & 7 \\
\hline Knox & Kirkpatrick Cave & KN62 & 2014: 9 Feb; 6 Jul & & 1 & & & & 2 & 3 \\
\hline Knox & Unreported Cave & KN90 & 2014: $5 \mathrm{Apr}$ & & 2 & & & 1 & 1 & 4 \\
\hline Knox & Brents Cave & KN112 & 2012: 8 May & & 1 & & & & 1 & 2 \\
\hline Knox & Burnett Cave & KN125 & 2008: 21 May & & 2 & & & & & 2 \\
\hline Knox & Chriscroft Cave & KN127 & 2014: 20 Oct & & 2 & & & 1 & 1 & 4 \\
\hline Knox & The Lost Puddle & KN145 & 2012: 8 May & & 2 & & & & 1 & 3 \\
\hline Knox & Ebenezer Rising Cave & KN150 & 2004: 20 Nov & 2 & 1 & & 1 & 2 & & 6 \\
\hline Knox & Meads River Cave & KN151 & $\begin{array}{l}\text { 2004: } 23 \text { Oct } \\
\text { 2007: } 22 \text { Apr; } \\
\text { 8, } 24 \text { Nov; } 2 \text { Dec }\end{array}$ & & 8 & 1 & & & 1 & 10 \\
\hline Knox & Fifth Entrance Cave & KN167 & $\begin{array}{l}\text { 2004: } 23 \text { Oct } \\
\text { 2007: } 8 \text { Nov }\end{array}$ & & 4 & & & & & 4 \\
\hline Knox & Aycock Spring Cave & KN172 & 2005: 17 Sep & 2 & 5 & & & & & 7 \\
\hline
\end{tabular}


Table 1. Continued.

\begin{tabular}{|c|c|c|c|c|c|c|c|c|c|}
\hline County & Cave & TCS No. & Date & $\mathrm{F}$ & A $\mathrm{R}$ & $\mathrm{B}$ & NM & Bats & Total \\
\hline Knox & Steamboat Crawl & KN173 & 2007: 5 Apr & & 3 & & & & 3 \\
\hline Loudon & Blankenship Cave & LN1 & 2014: 25 Jan & & 1 & & & 1 & 2 \\
\hline Loudon & Benjos Cave & LN11 & 2014: 30 Aug & & 2 & & & & 2 \\
\hline Loudon & Phantom Insurgence Cave & LN22 & 2014: 30 Aug & 2 & 5 & & 1 & 1 & 9 \\
\hline McMinn & Small Cave & MM5 & 2014: 10 May & & 6 & 1 & 2 & 2 & 11 \\
\hline McMinn & Too Small Cave & MM6 & 2014: 10 May & & 2 & 1 & 1 & & 4 \\
\hline Meigs & Sensabaugh Cave & ME3 & 2014: 31 Aug & & 3 & & 1 & 1 & 5 \\
\hline Monroe & The Lost Sea & MO1 & 2014: 9 Sep & & & & & & 0 \\
\hline Monroe & Gay Cave & MO3 & 2013: 16 Nov & & 2 & & 2 & 1 & 5 \\
\hline Monroe & Morgan Cave & MO5 & 2013: 26 Oct & & 3 & & 2 & 1 & 6 \\
\hline Monroe & Nobletts Cave & MO6 & 2014: $26 \mathrm{Nov}$ & & 2 & & 1 & 1 & 4 \\
\hline Monroe & Lick Creek Cave & MO8 & 2013: 16 Nov & 3 & & 1 & 1 & 1 & 6 \\
\hline Monroe & Alans Hideway Cave & MO9 & 2013: 16 Nov & & 2 & 1 & & 1 & 4 \\
\hline Rhea & Grassy Creek Cave & RH2 & 2014: 22 Dec & & 3 & & 1 & 2 & 6 \\
\hline Roane & Berry Cave & RN3 & $\begin{array}{l}\text { 2004: } 17 \text { Dec } \\
\text { 2005: } 5 \text { Mar } \\
\text { 2014: } 28 \text { Jun }\end{array}$ & 3 & 5 & 1 & 1 & 2 & 12 \\
\hline Roane & Cave Creek Cave & RN5 & $\begin{array}{l}\text { 2007: } 7 \text { Jun } \\
\text { 2014: } 28 \text { Jun }\end{array}$ & 2 & 4 & 1 & 1 & 1 & 9 \\
\hline Roane & Eblen Cave & RN6 & $\begin{array}{l}\text { 2005: } 30 \text { Dec } \\
\text { 2013: } 15 \text { May }\end{array}$ & 1 & 3 & & 2 & 2 & 8 \\
\hline Roane & Big Cave & RN13 & 2005: 5 Mar & & 2 & & & 2 & 4 \\
\hline Roane & Chimney Cave & RN14 & 2005: 5 Mar & & & & & 1 & 1 \\
\hline Sevier & Two County Cave & SV36 & 2014: $5 \mathrm{Jul}$ & 1 & 1 & & 1 & & 3 \\
\hline Union & Oaks Cave & UN5 & 2015: 23 Mar & & 1 & 1 & 2 & 3 & 7 \\
\hline Union & Wright Cave & UN9 & 2015: $21 \mathrm{Mar}$ & & 2 & 1 & 1 & 1 & 5 \\
\hline Union & Big Cave & UN10 & 2015: 22 Mar & & 3 & 1 & 2 & & 6 \\
\hline Union & Rogers Hollow Cave & UN23 & 2015: 22 Mar & & 1 & 1 & 1 & 1 & 4 \\
\hline Union & Mossy Spring Cave & UN25 & 2015: 22 Mar & & 3 & & 2 & & 5 \\
\hline Union & Ellison Hollow Cave & UN46 & 2015: 22 Mar & & 1 & 1 & & & 2 \\
\hline
\end{tabular}

Two additional ranks associated with extinction exist: GH (Possibly Extinct) and GX (Presumed Extinct). At the global scale, a Questionable rank qualifier (Q) can be used to denote uncertainty in the conservation status rank (e.g., G2Q). Status ranks are assessed at three geographic scales: global (G1-5), national (N1-5), and state (S1-5). Ranks at the global and state scales are given in the text and Table 2.

\section{RESUlts AND Discussion}

Annotated List of Vertebrate Fauna of the

Appalachians Karst Region in Eastern Tennessee

Phylum Chordata

Subphylum Vertebrata

Class Actinopterygii

Order Cypriniformes

Family Cyprinidae

Genus Luxilus

Luxilus chrysocephalus Rafinesque, 1820 (AC) Striped Shiner
Localities: Rhea Co.: Grassy Creek Cave (RH2)*.

Conservation status: IUCN: Least Concern; NatureServe: G5 (S5 in Tennessee).

Comments: An adult was observed in the main stream near the upstream quarry entrance of Grassy Creek Cave. This represents the first report of this species from a cave. We hypothesize that fish enter caves either from being washed in during flooding events or following streams upstream and entering caves through springs, which may be the situation for Grassy Creek Cave.

References: * present study.

\section{Genus Nocomis}

Nocomis micropogon (Cope, 1865) (AC) River Chub

Localities: Monroe Co.: Lick Creek Cave (MO8)*, karst window NNE of Lick Creek Cave*.

Conservation status: IUCN: Least Concern; NatureServe: G5 (S5 in Tennessee).

Comments: A single fish was observed in the sump pool of Lick Creek Cave. Three fish were observed in a shallow pool at the base of a karst window ca. $250 \mathrm{~m} \mathrm{NNE}$ of the 


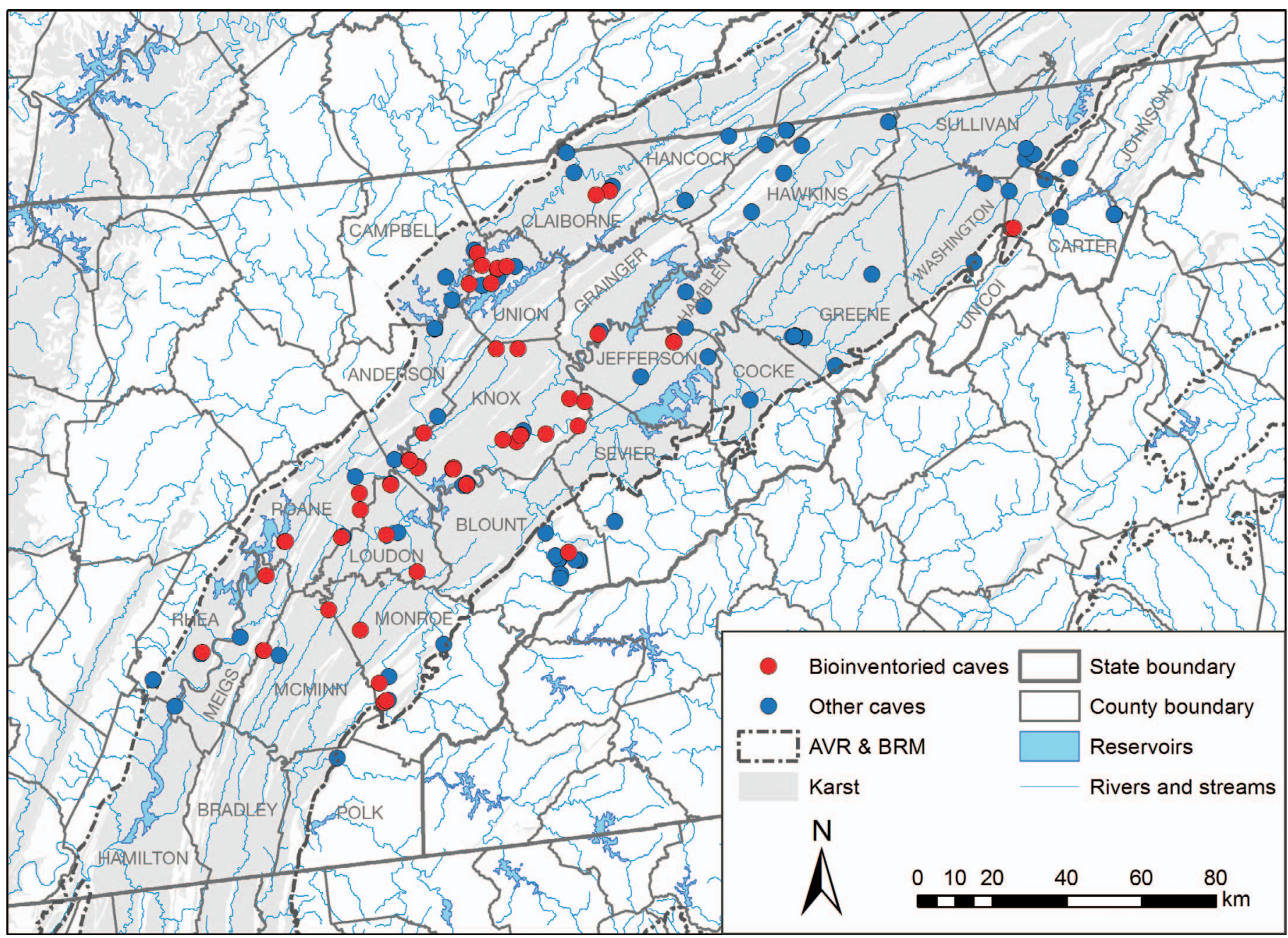

Figure 1. Map of 56 caves bioinventoried and 76 additional caves with vertebrate records in the Appalachian Valley and Ridge and adjacent Blue Ridge Mountains of eastern Tennessee from 24 counties. Karst carbonate rock are depicted in gray based on the U.S. Karst Map (Weary and Doctor, 2014).

spring entrance to Lick Creek Cave. This species was previously reported from caves in West Virginia (Poly and Boucher, 1996).

References: * present study.

\section{Genus Notropis}

Notropis spp. (AC) Unidentified shiner

Localities: Knox Co.: Christian Cave (KN49)*, Ebenezer Rising Cave (KN150)*, Aycock Spring Cave (KN172)*; Loudon Co.: Phantom Insurgence Cave (LN22)*; Rhea Co.: Grassy Creek Cave (RH2)*; Roane Co.: Berry Cave (RN3)*, Cave Creek Cave (RN5)*.

Conservation status: Not applicable.

Comments: Shiners were observed but could not be captured to confirm species identity. Several Notropis species have been reported from caves in the eastern United States (Bailey, 1933; Kuehne, 1966; Armstrong and Williams, 1971; Relyea and Sutton, 1973; Pearson and Boston, 1995; Poly and Boucher, 1996; Poly, 2001; Ruhl,
2005) and may wash into caves during flooding events and become trapped after waters recede. Fifteen Notropis species are known from the Appalachian Valley and Ridge of eastern Tennessee (Etnier and Starnes, 1993), and several genera of Cyprinidae have been reported from caves in nearby regions of northern Alabama (Rheams et al., 1992), including Notropis minnows. It is possible that cave systems may allow some aquatic surface species to disperse across hydrological drainage divides (Ray et al., 2014).

References: * present study.

\section{Genus Rhinichthys}

Rhinichthys atratulus (Hermann, 1804) (AC) Eastern Blacknose Dace

Localities: Claiborne Co.: a cave near New Tazewell ${ }^{1}$.

Conservation status: IUCN: Least Concern; NatureServe: G5 (S5 in Tennessee).

Comments: Evermann and Hildebrand (1914) collected eight specimens from a cave near New Tazewell in October 
1893. This species has also been reported from caves in West Virginia (Reese, 1934; Dearolf, 1956; Poly and Boucher, 1996; Poly, 2001).

References: ${ }^{1}$ Evermann and Hildebrand (1914).

\section{Order Perciformes \\ Family Centrarchidae \\ Genus Lepomis}

Lepomis cyanellus Rafinesque, 1819 (AC) Green Sunfish

Localities: Loudon Co.: Phantom Insurgence Cave (LN22)*; Roane Co.: Berry Cave (RN3)*.

Conservation status: IUCN: Least Concern; NatureServe: G5 (S5 in Tennessee).

Comments: This sunfish has been reported from several caves in the eastern United States (Jones and Hettler, 1959; McDaniel and Gardner, 1977; Pearson and Boston, 1995; Poly and Boucher, 1996; Poly, 2001; Ruhl, 2005).

References: * present study.

Lepomis macrochirus Rafinesque, 1819 (AC) Bluegill

Localities: Knox Co.: Ebenezer Rising Cave (KN150)*; Monroe Co.: Lick Creek Cave (MO8)*; Rhea Co.: Grassy Creek Cave (RH2)*; Roane Co.: Berry Cave (RN3)*.

Conservation status: IUCN: Least Concern; NatureServe: G5 (S5 in Tennessee).

Comments: This species has been reported from caves in several states in the eastern United States (Franz et al., 1994; Pearson and Boston, 1995; Poly and Boucher, 1996; Lewis, 1998; Poly, 2001; Ruhl, 2005).

References: * present study.

\section{Order Scorpaeniformes \\ Family Cottidae \\ Genus Cottus}

Cottus carolinae (Gill, 1861) (SP) Banded Sculpin

Localities: Grainger Co.: Indian Cave (GA4)*; Knox Co.: Christian Cave (KN49)*, Aycock Spring Cave (KN172)*; Monroe Co.: Lick Creek Cave (MO8)*; Roane Co.: Cave Creek Cave (RN5)*, Eblen Cave (RN6)*; Sevier Co.: Two County Cave (SV36)*.

Conservation status: IUCN: Least Concern; NatureServe: G5 (S5 in Tennessee).

Comments: Cottus carolinae is the most commonly observed fish in caves of the Interior Low Plateau and Appalachian Valley (Cope and Packard, 1881; Bailey, 1933; Dearolf, 1956; Pearson and Boston, 1995; Poly and Boucher, 1996; Ruhl, 2005; Niemiller et al., 2006). Many populations likely live year-round in caves and some populations are noted as possessing troglomorphic traits (Espinasa and Jeffery, 2003; Espinasa et al., 2013). Although common in several eastern Tennessee caves, no populations in this region are known to be troglomorphic.

References: * present study.

\section{Order Siluriformes \\ Family Ictaluridae \\ Genus Ameiurus}

Ameiurus natalis (Lesueur, 1819) (SX/AC) Yellow Bullhead
Localities: Knox Co.: Mudflats Cave (KN9)*.

Conservation status: IUCN: Least Concern; NatureServe: G5 (S5 in Tennessee).

Comments: This species is known from caves in Alabama, Florida, Kentucky, and Mississippi (Armstrong and Williams, 1971; Relyea and Sutton, 1973; Cliburn and Middleton, 1983; Franz et al., 1994; Pearson and Boston, 1995; Poly, 2001). This is the first report from a cave in eastern Tennessee.

References: * present study.

\section{Class Amphibia \\ Order Anura \\ Family Bufonidae \\ Genus Anaxyrus}

Anaxyrus americanus (Holbrook, 1836) (AC) American

Toad

Localities: Blount Co.: Gregory Cave (BA4) ${ }^{1}$.

Conservation status: IUCN: Least Concern; NatureServe: G5 (S5 in Tennessee).

Comments: Dodd et al. (2001) report this toad from the entrance of Gregory Cave in Cades Cove, Great Smoky Mountains National Park.

References: ${ }^{1}$ Dodd et al. (2001).

Anaxyrus fowleri (Hinckley, 1882) (AC) Fowler's Toad

Localities: Blount Co.: Gregory Cave (BA4) ${ }^{1}$.

Conservation status: IUCN: Least Concern; NatureServe: G5 (S5 in Tennessee).

Comments: Dodd et al. (2001) report this toad from the entrance of Gregory Cave in Cades Cove, Great Smoky Mountains National Park.

References: ${ }^{1}$ Dodd et al. (2001).

Family Hylidae

Genus Pseudacris

Pseudacris crucifer (Wied-Neuwied, 1838) (AC) Spring Peeper

Localities: Knox Co.: Meads River Cave (KN151)*.

Conservation status: IUCN: Least Concern; NatureServe: G5 (S5 in Tennessee).

Comments: This treefrog has been infrequently reported from caves (Black, 1971; Prather and Briggler, 2001; Godwin, 2008; Niemiller and Miller, 2009). Most of these records are thought to represent accidental occurrences, but $P$. crucifer may seek refuge in small caves during drought conditions (Prather and Briggler, 2001).

References: * present study.

\section{Pseudacris feriarum (Baird, 1854) (AC) Upland Chorus} Frog

Localities: Knox Co.: Steamboat Crawl (KN173)*.

Conservation status: IUCN: Least Concern; NatureServe: G5 (S5 in Tennessee).

Comments: This treefrog has been infrequently reported from caves (Black, 1971; Osbourn, 2005; Godwin, 2008; Niemiller and Miller, 2009).

References: * present study.

Journal of Cave and Karst Studies, April 2016•7 
Table 2. Ecological classification, conservation status, and number of eastern Tennessee caves and counties from which species are documented by bioinventories of 56 caves (Current) and from the literature, museum, and database sources for other caves (Other).

\begin{tabular}{|c|c|c|c|c|c|c|c|c|}
\hline Species & $\begin{array}{c}\text { Ecological } \\
\text { Classification }\end{array}$ & $\begin{array}{l}\text { IUCN } \\
\text { Red List }\end{array}$ & $\begin{array}{c}\text { NatureServe } \\
\text { Status }\end{array}$ & $\begin{array}{c}\text { Gov } \\
\text { Status }\end{array}$ & $\begin{array}{l}\text { Caves } \\
\text { Current }\end{array}$ & $\begin{array}{l}\text { Caves } \\
\text { Other }\end{array}$ & $\begin{array}{l}\text { Caves } \\
\text { Total }\end{array}$ & Counties \\
\hline \multicolumn{9}{|l|}{ Fishes } \\
\hline Luxilus chrysocephalus & $\mathrm{AC}$ & $\mathrm{LC}$ & G5, S5 & & 1 & 0 & 1 & 1 \\
\hline Nocomis micropogon & $\mathrm{AC}$ & $\mathrm{LC}$ & G5, S5 & & 1 & 0 & 1 & 1 \\
\hline Notropis spp. & $\mathrm{AC}$ & & & & 7 & 0 & 7 & 4 \\
\hline Rhinichthys atratulus & $\mathrm{AC}$ & $\mathrm{LC}$ & G5, S5 & & 0 & 1 & 1 & 1 \\
\hline Lepomis cyanellus & $\mathrm{AC}$ & $\mathrm{LC}$ & G5, S5 & & 2 & 0 & 2 & 2 \\
\hline Lepomis macrochirus & $\mathrm{AC}$ & $\mathrm{LC}$ & G5, S5 & & 4 & 0 & 4 & 4 \\
\hline Cottus carolinae & SP & $\mathrm{LC}$ & G5, S5 & & 7 & 0 & 7 & 5 \\
\hline Ameiurus natalis & SX/AC & $\mathrm{LC}$ & G5, S5 & & 1 & 0 & 1 & 1 \\
\hline \multicolumn{9}{|l|}{ Amphibians (Frogs and Toads) } \\
\hline Anaxyrus americanus & $\mathrm{AC}$ & $\mathrm{LC}$ & G5, S5 & & 0 & 1 & 1 & 1 \\
\hline Anaxyrus fowleri & $\mathrm{AC}$ & $\mathrm{LC}$ & G5, S5 & & 0 & 1 & 1 & 1 \\
\hline Pseudacris crucifer & $\mathrm{AC}$ & $\mathrm{LC}$ & G5, S5 & & 1 & 0 & 1 & 1 \\
\hline Pseudacris feriarum & $\mathrm{AC}$ & $\mathrm{LC}$ & G5, S5 & & 1 & 0 & 1 & 1 \\
\hline Lithobates catesbeianus & TX & $\mathrm{LC}$ & G5, S5 & & 4 & 0 & 4 & 2 \\
\hline Lithobates clamitans & TX & $\mathrm{LC}$ & G5, S5 & & 9 & 1 & 10 & 7 \\
\hline Lithobates palustris & $\mathrm{TX}$ & $\mathrm{LC}$ & G5, S5 & & 11 & 2 & 13 & 7 \\
\hline Lithobates sylvaticus & TX/AC & $\mathrm{LC}$ & G5, S5 & & 0 & 1 & 1 & 1 \\
\hline \multicolumn{9}{|l|}{ Amphibians (Salamanders) } \\
\hline Desmognathus conanti & $\mathrm{AC}$ & $\mathrm{LC}$ & G5, S5 & & 1 & 1 & 2 & 2 \\
\hline Desmognathus quadramaculatus & $\mathrm{AC}$ & $\mathrm{LC}$ & G5, S4 & & 0 & 1 & 1 & 1 \\
\hline Eurycea cirrigera & $\mathrm{TX}$ & $\mathrm{LC}$ & G5, S5 & & 1 & 0 & 1 & 1 \\
\hline Eurycea longicauda & TP/TX & $\mathrm{LC}$ & G5, S5 & & 4 & 3 & 7 & 6 \\
\hline Euryea lucifuga & $\mathrm{TP}$ & $\mathrm{LC}$ & G5, S5 & & 38 & 14 & 48 & 19 \\
\hline Eurycea wilderae & $\mathrm{AC}$ & $\mathrm{LC}$ & G5, S5 & & 2 & 2 & 4 & 2 \\
\hline Gyrinophilus gulolineatus & SB & EN & G1Q, S1 & FC, ST & 9 & 9 & 11 & 4 \\
\hline Gyrinophilus porphyriticus & TP & $\mathrm{LC}$ & G5, S5 & & 18 & 12 & 25 & 11 \\
\hline Plethodon dorsalis & TP/TX & $\mathrm{LC}$ & G5, S4 & & 9 & 2 & 10 & 3 \\
\hline Plethodon glutinosus & $\mathrm{TP}$ & $\mathrm{LC}$ & G5, S5 & & 19 & 13 & 31 & 11 \\
\hline Pseudotriton ruber & $\mathrm{TP}$ & $\mathrm{LC}$ & G5, S5 & & 3 & 1 & 4 & 3 \\
\hline \multicolumn{9}{|l|}{ Reptiles (Snakes) } \\
\hline Diadophis punctatus & $\mathrm{AC}$ & $\mathrm{LC}$ & G5, S5 & & 1 & 0 & 1 & 1 \\
\hline Lampropeltis nigra & $\mathrm{AC}$ & $\mathrm{LC}$ & G5, S5 & & 0 & 1 & 1 & 1 \\
\hline Pantherophis spiloides & $\mathrm{AC}$ & $\mathrm{LC}$ & G5, S5 & & 0 & 1 & 1 & 1 \\
\hline Thamnophis sirtalis & $\mathrm{AC}$ & $\mathrm{LC}$ & G5, S5 & & 1 & 0 & 1 & 1 \\
\hline Agkistrodon contortrix & $\mathrm{AC}$ & $\mathrm{LC}$ & G5, S5 & & 0 & 1 & 1 & 1 \\
\hline \multicolumn{9}{|l|}{ Reptiles (Turtles) } \\
\hline Terrapene carolina & $\mathrm{AC}$ & VU & G5, S4 & & 2 & 0 & 2 & 2 \\
\hline \multicolumn{9}{|l|}{ Birds } \\
\hline Cathartes aura & TX/AC & $\mathrm{LC}$ & G5, S5 & & 0 & 1 & 1 & 1 \\
\hline Coragyp satratus & TX/AC & $\mathrm{LC}$ & G5, S4 & & 0 & 4 & 4 & 2 \\
\hline Sayornis phoebe & $\mathrm{TX}$ & $\mathrm{LC}$ & G5, S5 & & 17 & 1 & 18 & 8 \\
\hline \multicolumn{9}{|l|}{ Mammals (Bats) } \\
\hline Corynorhinus rafinesquii & $\mathrm{TX}$ & $\mathrm{LC}$ & $\mathrm{G} 3 \mathrm{G} 4, \mathrm{~S} 3$ & & 0 & 11 & 11 & 6 \\
\hline Eptesicus fuscus & $\mathrm{TX}$ & $\mathrm{LC}$ & $\mathrm{G} 5, \mathrm{~S} 5$ & & 10 & 34 & 41 & 16 \\
\hline Lasiurus borealis & $\mathrm{AC}$ & $\mathrm{LC}$ & $\mathrm{G} 4, \mathrm{~S} 5$ & & 0 & 2 & 2 & 1 \\
\hline Lasiurus cinereus & $\mathrm{TX} / \mathrm{AC}$ & $\mathrm{LC}$ & G4, S5 & & 0 & 2 & 2 & 2 \\
\hline Myotis grisescens & $\mathrm{TX}$ & NT & $\mathrm{G} 3, \mathrm{~S} 2$ & FE, SE & 6 & 35 & 37 & 17 \\
\hline Myotis leibii & $\mathrm{TX}$ & $\mathrm{LC}$ & $\mathrm{G} 3 \mathrm{G} 4, \mathrm{~S} 2 \mathrm{~S} 3$ & $\mathrm{SD}$ & 0 & 6 & 6 & 4 \\
\hline Myotis lucifugus & TX & $\mathrm{LC}$ & G3, S5 & & 3 & 26 & 29 & 12 \\
\hline
\end{tabular}

8• Journal of Cave and Karst Studies, April 2016 
Table 2. Continued.

\begin{tabular}{|c|c|c|c|c|c|c|c|c|}
\hline Species & $\begin{array}{c}\text { Ecological } \\
\text { Classification }\end{array}$ & $\begin{array}{c}\text { IUCN } \\
\text { Red List }\end{array}$ & $\begin{array}{c}\text { NatureServe } \\
\text { Status }\end{array}$ & $\begin{array}{l}\text { Gov } \\
\text { Status }\end{array}$ & $\begin{array}{l}\text { Caves } \\
\text { Current }\end{array}$ & $\begin{array}{l}\text { Caves } \\
\text { Other }\end{array}$ & $\begin{array}{l}\text { Caves } \\
\text { Total }\end{array}$ & Counties \\
\hline Myotis septentrionalis & TX & $\mathrm{LC}$ & $\mathrm{G} 1 \mathrm{G} 2, \mathrm{~S} 4$ & FT & 1 & 21 & 21 & 10 \\
\hline Myotis sodalis & TX & EN & $\mathrm{G} 2, \mathrm{~S} 1$ & FE, SE & 0 & 13 & 13 & 6 \\
\hline Perimyotis subflavus & TX & $\mathrm{LC}$ & G3, S5 & & 37 & 63 & 89 & 22 \\
\hline \multicolumn{9}{|l|}{ Mammals (non-Bats) } \\
\hline Canis latrans & TX/AC & $\mathrm{LC}$ & G5, S5 & & 3 & 0 & 3 & 2 \\
\hline Procyon lotor & $\mathrm{TX}$ & $\mathrm{LC}$ & G5, S5 & & 27 & 2 & 29 & 12 \\
\hline Castor canadensis & TX & $\mathrm{LC}$ & G5, S5 & & 2 & 0 & 2 & 2 \\
\hline Neotoma magister & TX & NT & G3G4, S3 & SD & 7 & 6 & 13 & 7 \\
\hline Peromyscus gossypinus & TX & $\mathrm{LC}$ & G5, S5 & & 0 & 1 & 1 & 1 \\
\hline Peromyscus leисориs & TX & $\mathrm{LC}$ & G5, S5 & & 2 & 0 & 2 & 2 \\
\hline Peromyscus sp. & TX/AC & & & & 2 & 0 & 2 & 2 \\
\hline Blarina brevicauda & $\mathrm{AC}$ & $\mathrm{LC}$ & G5, S5 & & 1 & 0 & 1 & 1 \\
\hline
\end{tabular}

Note: FE $=$ Federally Endangered, FT $=$ Federally Threatened, FC $=$ Federal Candidate Species, $\mathrm{SE}=$ State Endangered, ST $=$ State Threatened, and SD $=$ State Deemed in Need of Management.

\section{Family Ranidae}

Genus Lithobates

Lithobates catesbeianus (Shaw, 1802) (TX)

American Bullfrog

Localities: Knox Co.: Cruze Cave (KN24)*, Meads Quarry Cave (KN28)*, Meads River Cave (KN151)*; McMinn Co.: Small Cave (MM5)*.

Conservation status: IUCN: Least Concern; NatureServe: G5 (S5 in Tennessee).

Comments: This species and L. clamitans are frequently found in caves with aquatic habitat, particularly near entrances. It has been reported from several caves in Tennessee (Barr, 1953; Lewis, 2005; Niemiller and Miller, 2009).

References: * present study.

\section{Lithobates clamitans (Rafinesque, 1820) (TX) Green Frog}

Localities: Blount Co.: Gregory Cave (BA4) ${ }^{1}$; Grainger Co.: Indian Cave (GA4)*; Knox Co.: Meads Quarry Cave (KN28)*, Meads River Cave (KN151)*, Fifth Entrance Cave (KN167)*, Aycock Spring Cave (KN172)*; Loudon Co.: Phantom Insurgence Cave (LN22)*; Monroe Co.: Alans Hideaway Cave (MO9)*; Roane Co.: Berry Cave (RN3)*; Sevier Co.: Two County Cave (SV36)*.

Conservation status: IUCN: Least Concern; NatureServe: G5 (S5 in Tennessee).

Comments: Lithobates clamitans has been reported from several caves in Tennessee (Barr, 1953; Dodd et al., 2001; Lewis, 2005; Niemiller and Miller, 2005, 2009).

References: ${ }^{1}$ Dodd et al. (2001); * present study.

\section{Lithobates palustris (LeConte, 1825) (TX) Pickerel Frog} Localities: Blount Co.: Gregory Cave (BA4) ${ }^{2}$; Campbell Co.: Panther Cave No. 1 (CM8)*; Knox Co: Christian Cave (KN49)*, Ebenezer Rising Cave (KN150)*, Meads River Cave (KN151)*, Fifth Entrance Cave (KN167)*, Aycock Spring Cave (KN172)*; Loudon Co.: Phantom
Insurgence Cave (LN22)*; McMinn Co.: Small Cave (MM5) $^{1}$; Meigs Co.: Sensabaugh Cave (ME3)*; Roane Co.: Berry Cave (RN3)*, Cave Creek Cave (RN5)*, Eblen Cave (RN6)*.

Conservation status: IUCN: Least Concern; NatureServe: G5 (S5 in Tennessee).

Comments: This species is commonly found in caves with streams in entrance and twilight areas (Cliburn and Middleton, 1983; Garton et al., 1993; Buhlmann, 2001; Camp and Jensen, 2007; Niemiller and Miller, 2009).

References: VertNet: ${ }^{1}$ NCSM; ${ }^{2}$ Dodd et al. (2001); * present study.

\section{Lithobates sylvaticus (LeConte, 1825) (TX/AC) Wood Frog} Localities: Blount Co.: Gregory Cave (BA4) ${ }^{1}$.

Conservation status: IUCN: Least Concern; NatureServe: G5 (S5 in Tennessee).

Comments: Dodd et al. (2001) report this frog from the entrance of Gregory Cave in Cades Cove, Great Smoky Mountains National Park.

References: ${ }^{1}$ Dodd et al. (2001).

\section{Order Caudata \\ Family Plethodontidae \\ Genus Desmognathus}

Desmognathus conanti Rossman, 1958 (TX) Spotted Dusky Salamander

Localities: Blount Co.: Whiteoak Blowhole Cave (BA2) $^{1,2}$; Knox Co.: Burnett Cave (KN125)*.

Conservation status: IUCN: Least Concern; NatureServe: G5 (S5 in Tennessee).

Comments: This species is found in a variety of aquatic habitats in forest areas, including springs, seeps, spring runs, and small- to medium-sized streams (Wyckoff and Niemiller, 2011). Although it occasionally can be found in and around the entrances of caves that issue at the surface 
as springs in Tennessee, it is seldom observed in the dark zone (Niemiller and Miller, 2009).

References: ${ }^{1,2}$ Wallace $(1984,2003) ;{ }^{*}$ present study.

\section{Desmognathus quadramaculatus}

(Holbrook, 1840) (AC) Black-Bellied Salamander

Localities: Blount Co.: Rainbow Cave (BA26) ${ }^{1,2}$.

Conservation status: IUCN: Least Concern; NatureServe: G5 (S4 in Tennessee).

Comments: Wallace $(1984,2003)$ observed one individual at the entrance to Rainbow Cave in Great Smoky Mountain National Park.

References: ${ }^{1,2}$ Wallace $(1984,2003)$.

\section{Genus Eurycea}

Eurycea cirrigera (Green, 1818) (TX) Southern Two-Lined Salamander

Localities: Loudon Co.: Phantom Insurgence Cave (LN22)*.

Conservation status: IUCN: Least Concern; NatureServe: G5 (S5 in Tennessee).

Comments: This salamander has been infrequently reported from caves in Tennessee (Lewis, 2005; Niemiller and Miller, 2007, 2009), as well as northwestern Georgia (Camp and Jensen, 2007). This is the first report from the Valley and Ridge of eastern Tennessee.

References: * present study.

\section{Eurycea longicauda (Green, 1818) (TP/TX) Long-Tailed Salamander}

Localities: Blount Co.: Gregory Cave (BA4) ${ }^{1-4}$, Tory Shields Bluff Cave (BA56) ${ }^{1,2}$; Campbell Co.: Panther Cave No. 1 (CM8)*; Knox Co.: Meads Quarry Cave (KN28)*; McMinn Co.: Small Cave (MM5)*; Monroe Co.: Morgan Cave (MO5)*; Sevier Co.: Stupkas Cave (SV42) ${ }^{2}$.

Conservation status: IUCN: Least Concern; NatureServe: G5 (S5 in Tennessee).

Comments: This species is a common inhabitant of caves in the Interior Low Plateau and Appalachian Valley (Garton et al., 1993; Buhlmann, 2001; Dodd et al., 2001; Lewis, 2005; Osbourn, 2005; Camp and Jensen, 2007; Niemiller and Miller, 2009). It is known to reproduce in caves in Tennessee (Dodd et al., 2001; Taylor and Mays, 2006).

References: ${ }^{1,2}$ Wallace $(1984,2003) ;{ }^{3}$ Dodd et al. (2001); ${ }^{4}$ Taylor and Mays (2006); * present study.

\section{Eurycea lucifuga Rafinesque, 1822 (TP) Cave Salamander}

Localities: Anderson Co.: Norris Dam Cave No. 2 $(\mathrm{AN} 32)^{2}$; Blount Co.: Tuckaleechee Caverns (BA11) ${ }^{5,7, *}$, Calf Cave No. 2 (BA20) ${ }^{5,7}$; Campbell Co.: Panther Cave No. 1 (CM8)*; Carter Co.: Carter Saltpeter Cave (CR1)*; Claiborne Co.: Sour Kraut Cave (CB46)*, Kings Saltpeter Cave (CB52)*; Grainger Co.: Indian Cave (GA4) ${ }^{2, *}$; Hancock Co.: Goodmans Cave (HN5) ${ }^{6}$; Jefferson Co.: Mutton Hollow Cave (JF41) ${ }^{4}$, Silo Pit Cave (JF71)*; Knox Co.: Mudflats Cave (KN9)*, Carter Cave (KN14)*, Keller Bend Cave (KN16)*, Blowing Hole Cave (KN19)*, Cruze Cave (KN24)*, Meads Quarry Cave $(\mathrm{KN} 28)^{2,10, *}$, Chris- tian Cave (KN49)*, Keller Bluff Cave No. 1 (KN61) ${ }^{3}$, Unreported Cave (KN90)*, Brents Cave (KN112)*, Chriscroft Cave (KN127)*, The Lost Puddle (KN145)*, Meads River Cave (KN151)*, Fifth Entrance Cave (KN167)*, Steamboat Crawl (KN173)*; Loudon Co.: Blankenship Cave (LN1)*, Benjos Cave (LN11)*, Phantom Insurgence Cave (LN22)*; McMinn Co.: Small Cave (MM5)*, Too Small Cave (MM6)*; Meigs Co.: Sensabaugh Cave (ME3)*; Monroe Co.: Morgan Cave (MO5)*, Nobletts Cave (MO6) ${ }^{6, *}$; Rhea Co.: Grassy Creek Cave $(\mathrm{RH} 2)^{6}$, Marler Cave (RH4) ${ }^{9}$; Roane Co.: Berry Cave (RN3)*, Cave Creek Cave (RN5)*, Eblen Cave (RN6)*, Big Cave (RN13)*; Sevier Co.: Stupkas Cave (SV42), ${ }^{5,7,}$; Sullivan Co.: Morrell Cave (SL6) ${ }^{1}$; Union Co.: Wright Cave (UN9)*, Big Cave (UN10)*, Rogers Hollow Cave (UN23)*, Mossy Spring Cave (UN25)*, Ellison Hollow Cave (UN46); Washington Co.: Keplinger Cave (WS3) ${ }^{3}$.

Conservation status: IUCN: Least Concern; NatureServe: G5 (S5 in Tennessee).

Comments: Eurycea lucifuga is the most common salamander found in caves of Tennessee (Niemiller and Miller, 2009). Niemiller et al. (2009a) report on reproduction of this species from Meads Quarry Cave. A female and eight hatchlings with yolk sacs were found in a rimstone pool in Blankenship Cave (LN1) on 25 January 2014.

References: VertNet: ${ }^{1} \mathrm{CM},{ }^{2}$ USNM,${ }^{3} \mathrm{TNNH} ;{ }^{4}$ Ives (1951); 5,6,7 Wallace (1984, 1989, 2003); ${ }^{8}$ Dodd et al. (2001); ${ }^{9}$ Lewis (2005); ${ }^{10}$ Niemiller et al. (2009a); * present study.

\section{Eurycea wilderae Dunn, 1920 (AC) Blue Ridge Two-Lined Salamander}

Localities: Blount Co.: Whiteoak Blowhole Cave $(B A 2)^{1,2}$, Gregory Cave (BA4) ${ }^{3}$; Monroe Co.: Gay Cave (MO3)*, Alans Hideaway Cave (MO9)*.

Conservation status: IUCN: Least Concern; NatureServe: G5 (S5 in Tennessee).

Comments: This species has been found infrequently in caves, typically near entrances.

References: ${ }^{1,2}$ Wallace $(1984,2003){ }^{3}$ Dodd et al. (2001); * present study.

\section{Genus Gyrinophilus \\ Gyrinophilus gulolineatus Brandon, 1965 (SB) Berry Cave Salamander}

Localities: Knox Co.: Mudflats Cave (KN9) $)^{4-9,11,12, *}$, Meads Quarry Cave (KN28) $)^{1,4,7-9,11,12, *}$, Christian Cave (KN49) $)^{8,9,11,12, *}$, The Lost Puddle (KN145)*, Meads River Cave $(\mathrm{KN} 151)^{8,12, *}$, Fifth Entrance Cave $(\mathrm{KN} 167)^{8,12, *}$, Aycock Spring Cave (KN172) ${ }^{8,9,11,12, *}$; McMinn Co.: Small Cave (MM5)*, roadside ditch near Oostanaula Creek south of Athens ${ }^{2-4,7-10,12}$; Meigs Co.: Blythe Ferry Cave (ME1),12; Roane Co.: Berry Cave (RN3, typelocality $)^{2-4,7-9,11,12, *}$.

Conservation status: IUCN: Endangered (B1ab(iii)+ 2ab(iii)); NatureServe: G1Q (S1 in Tennessee); Candidate 
for listing under the U.S. Endangered Species Act; listed as Threatened in Tennessee (Withers, 2009).

Comments: The records from The Lost Puddle and Small Cave represent new localities for this rare salamander.

References: VertNet: ${ }^{1}$ NCSM; ${ }^{2,3}$ Brandon (1965, 1966); ${ }^{4,5}$ Simmons $(1975,1976) ;{ }^{6}$ Caldwell and Copeland (1992); ${ }^{7,8}$ Miller and Niemiller $(2007,2008) ;{ }^{9,10}$ Niemiller and Miller (2010, 2011); ${ }^{11,12}$ Niemiller et al. $(2008,2010) ;$ * present study.

\section{Gyrinophilus porphyriticus (Green, 1827) (TP) Spring Salamander}

Localities: Blount Co.: Whiteoak Blowhole Cave $(B A 2)^{6,7}$; Rainbow Cave (BA26) ${ }^{8}$; Claiborne Co.: Buis Saltpeter Cave (CB48)*, Kings Saltpeter Cave (CB52)*; Grainger Co.: Indian Cave (GA4) ${ }^{2,3}$, ; Hawkins Co.: Pearson Cave $(\mathrm{HW} 12)^{1}$, Sensabaugh Saltpeter Cave $\left(\right.$ HW13) ${ }^{2}$; Knox Co.: Mudflats Cave (KN9) ${ }^{4,5}$, Carter Cave (KN14)*, Cruze Cave (KN24) ${ }^{9-11, *}$, Meads Quarry Cave (KN28) ${ }^{4, *}$, Kirkpatrick Cave (KN62)*, Burnett Cave (KN125)*, Meads River Cave (KN151) $^{9,10, *}$; Loudon Co.: Phantom Insurgence Cave (LN22)*; McMinn Co.: Small Cave (MM5)*, Wattenbarger Cave (MM9) ${ }^{2}$; Meigs Co.: Sensabaugh Cave (ME3)*; Monroe Co.: Nobletts Cave (MO6)*; Roane Co.: Cave Creek Cave (RN5) $^{9,10, *}$, Eblen Cave (RN6)*; Union Co.: Oaks Cave (UN5)*, Wolf Cave $(\mathrm{UN} 8)^{2,3}$, Big Cave (UN10)*, Mossy Spring Cave (UN25)*.

Conservation status: IUCN: Least Concern; NatureServe: G5 (S5 in Tennessee).

Comments: Gyrinophilus porphyriticus likely can be found in caves throughout the Appalachian Valley and Ridge province and Blue Ridge Mountains of eastern Tennessee. This species occurs syntopically and occasionally hybridizes with $G$. gulolineatus at Mudflats Cave, Meads Quarry Cave, and Meads River Cave in Knox County (Niemiller et al. 2008, 2009b). Both G. porphyriticus and G. gulolineatus were found at Small Cave in McMinn County.

References: VertNet: ${ }^{1} \mathrm{KU},{ }^{2} \mathrm{USNM} ;{ }^{3}$ Brandon (1966); ${ }^{4,5}$ Simmons $(1975,1976) ;{ }^{6,7}$ Wallace $(1984,2003) ;{ }^{8}$ Dodd et al. (2001); ${ }^{9,10}$ Miller and Niemiller (2007, 2008); ${ }^{11}$ Niemiller et al. (2008); * present study.

\section{Genus Plethodon \\ Plethodon dorsalis Cope, 1889 (TP/TX) Northern Zigzag Salamander}

Localities: Jefferson Co.: Tater Cave (JF8)*, Silo Pit Cave (JF71)*; Knox Co.: Mudflats Cave (KN9) ${ }^{1, *}$, Carter Cave (KN14)*, Blowing Hole Cave $(\mathrm{KN} 19)^{*}$, Meads Quarry Cave (KN28)*, Unreported Cave (KN90)*, Meads River Cave (KN151)*, Steamboat Crawl (KN173)*; Rhea Co.: Marler Cave (RH4) ${ }^{2}$.

Conservation status: IUCN: Least Concern; NatureServe: G5 (S4 in Tennessee).
Comments: This species is closely related to the Southern Zigzag Salamander ( $P$. ventralis); they are difficult to distinguish morphologically. Both species occur in caves in Tennessee (Lewis, 2005; Niemiller and Miller, 2009). Females are known to nest in small cavities in cave walls or crevices in clay floors (Miller et al., 1998; Niemiller and Miller, 2008).

References: ${ }^{1}$ Simmons (1975); ${ }^{2}$ Lewis (2005); * present study.

\section{Plethodon glutinosus (Green, 1818) (TP) Northern Slimy Salamander}

Localities: Blount Co.: Whiteoak Blowhole Cave $(\text { BA2) })^{3,5}$, Bull Cave (BA3) ${ }^{5}$, Gregory Cave (BA4) ${ }^{2,3,5,6,8}$, Calf Cave No. 1 (BA19) ${ }^{3,5,6}$, Calf Cave No. 2 (BA20) ${ }^{3,5}$, Rainbow Cave $(B A 26)^{6}$, Tory Shields Bluff Cave $\left(\right.$ BA56) ${ }^{3,5}$; Campbell Co.: Panther Cave No. 1 (CM8)*; Jefferson Co.: Tater Cave (JF8)*, Silo Pit Cave (JF71)*; Knox Co.: Mudflats Cave (KN9)*, Blowing Hole Cave (KN19)*, Cherokee Caverns (KN22)*, Cruze Cave $(\mathrm{KN} 24)^{2, *}$, Meads Quarry Cave (KN28)*, Chriscroft Cave (KN127)*, Aycock Spring Cave (KN172)*; Loudon Co.: Benjos Cave (LN11)*; McMinn Co.: Small Cave (MM5)*, Too Small Cave (MM6)*, Wattenbarger Cave (MM9) ${ }^{2}$; Monroe Co.: Gay Cave (MO3)*, Morgan Cave (MO5)*, Nobletts Cave (MO6) ${ }^{4}$, Double Sump Cave (MO13) ${ }^{1}$; Rhea Co.: Marler Cave (RH4) ${ }^{7}$; Roane Co.: Berry Cave (RN3)*, Cave Creek Cave (RN5)*, Big Cave (RN13)*; Sevier Co.: Stupkas Cave (SV42), ${ }^{3,5}$, Union Co.: Big Cave (UN10)*

Conservation status: IUCN: Least Concern; NatureServe: G5 (S5 in Tennessee).

Comments: This salamander is commonly encountered in caves throughout its range (Garton et al., 1993; Buhlmann, 2001; Dodd et al., 2001; Lewis, 2005; Taylor and Mays, 2006; Camp and Jensen, 2007; Godwin, 2008; Niemiller and Miller, 2009; Niemiller and Reeves, 2014). References: VertNet: ${ }^{1}$ CM, ${ }^{2}$ USNM; ${ }^{3,4,5}$ Wallace (1984, 1989, 2003); ${ }^{6}$ Dodd et al. (2001); ${ }^{7}$ Lewis (2005); ${ }^{8}$ Taylor and Mays (2006); * present study.

\section{Genus Pseudotriton \\ Pseudotriton ruber (Latreille, 1801) (TP) Red Salamander}

Localities: Blount Co.: Whiteoak Blowhole Cave $(\mathrm{BA} 2)^{1,2}$; Knox Co.: Aycock Spring Cave (KN172)*; Union Co.: Wright Cave (UN9)*, Mossy Spring Cave (UN25)*.

Conservation status: IUCN: Least Concern; NatureServe: G5 (S5 in Tennessee).

Comments: This species is frequently found in the twilight zone of caves with streams throughout the Interior Low Plateau and Appalachian Valley (Buhlmann, 2001; Osbourn, 2005; Camp and Jensen, 2007; Godwin, 2008; Miller et al., 2008; Niemiller and Miller, 2009; Niemiller and Reeves, 2014). Pseudotriton ruber utilizes cave streams and associated aquatic habitats for reproduction (Miller 
and Niemiller, 2005; Niemiller et al., 2006; Miller et al., 2008).

References: ${ }^{1,2}$ Wallace $(1984,2003) ;{ }^{*}$ present study.

\section{Class Reptilia \\ Order Squamata \\ Suborder Serpentes \\ Family Colubridae \\ Genus Diadophis}

Diadophis punctatus (Linneaus, 1766) (AC) Ring-Necked Snake

Localities: Knox Co.: Meads River Cave (KN151)*.

Conservation status: IUCN: Least Concern; NatureServe: G5 (S5 in Tennessee).

Comments: An adult was found crawling on a mud bank on 2 December 2007 in Meads River Cave (KN151) a few days after heavy rainfall. The snake was likely washed into the cave during flash flooding.

References: * present study.

\section{Genus Lampropeltis}

Lampropeltis nigra (Yarrow, 1882) (AC) Black Kingsnake

Localities: Sullivan Co.: Reagans Grapevine Cave (SL138) ${ }^{1}$.

Conservation status: IUCN: Least Concern; NatureServe: G5 (S5 in Tennessee).

Comments: Bailey (1988) reported seeing this species in February 1987.

References: ${ }^{1}$ Bailey (1988).

\section{Genus Pantherophis}

Pantherophis spiloides (Dumeril, Bibron, \& Dumeril, 1854)

(AC) Gray Ratsnake

Localities: Blount Co.: Gregory Cave (BA4) ${ }^{1}$.

Conservation status: IUCN: Least Concern; NatureServe: G5 (S5 in Tennessee).

Comments: Dodd et al. (2001) report this species from the entrance of Gregory Cave in Cades Cove, Great Smoky Mountains National Park.

References: ${ }^{1}$ Dodd et al. (2001).

\section{Family Natricidae \\ Genus Thamnophis}

Thamnophis sirtalis (Linnaeus, 1758) (AC) Common Gartersnake

Localities: Carter Co.: Carter Saltpeter Cave (CR1)*.

Conservation status: IUCN: Least Concern; NatureServe: G5 (S5 in Tennessee).

Comments: An adult was observed at the bottom of the large entrance sink in the dark zone on 14 May 2014, likely as a consequence of accidentally falling into the cave.

References: * present study.

\section{Family Viperidae \\ Genus Agkistrodon}

Agkistrodon contortrix (Linnaeus, 1766) (AC) Copperhead

Localities: Blount Co.: Gregory Cave (BA4) ${ }^{1}$.
Conservation status: IUCN: Least Concern; NatureServe: G5 (S5 in Tennessee).

Comments: Dodd et al. (2001) report this species from the entrance of Gregory Cave in Cades Cove, Great Smoky Mountains National Park.

References: ${ }^{1}$ Dodd et al. (2001).

\section{Order Testudines \\ Family Emydidae \\ Genus Terrapene}

Terrapene carolina (Linnaeus, 1758) (AC) Eastern Box Turtle

Localities: Jefferson Co.: Silo Pit Cave (JF71)*; Knox Co.: Meads Quarry Cave (KN28)*.

Conservation status: IUCN: Vulnerable; NatureServe: G5 (S4 in Tennessee).

Comments: An adult male was observed just inside the main entrance of Meads Quarry Cave on 4 November 2006. An adult male with a cracked carapace also was observed in the twilight zone at Silo Pit Cave on 3 Aug 2015. This species commonly falls into pits or gets washed into caves during flooding.

References: * present study.

\section{Class Aves \\ Order Cathartiformes \\ Family Cathartidae \\ Genus Cathartes}

Cathartes aura (Linnaeus, 1958) (TX/AC) Turkey Vulture

Localities: Washington Co.: unknown cave ${ }^{1}$.

Conservation status: IUCN: Least Concern; NatureServe: G5 (S5 in Tennessee).

Comments: This species is known to nest at the entrances and within the twilight zone of caves, particularly those occurring in bluffs (Coles, 1944; Lewis, 2005).

\section{References: VertNet: ${ }^{1} \mathrm{SBMNH}$.}

\section{Genus Coragyps}

Coragyps atratus (Bechstein, 1793) (TX/AC) Black Vulture

Localities: Sullivan Co.: cave in Slaughter Bluff on Holston River ${ }^{1}$, cave on south fork of Holston River ${ }^{2}$; Washington Co.: cave near Johnson City ${ }^{1}$; cave on Watauga River NW of Johnson City ${ }^{3}$.

Conservation status: IUCN: Least Concern; NatureServe: G5 (S4 in Tennessee).

Comments: Like Cathartes aura, this species is known to nest at the entrances and within the twilight zone of caves (Lyle, 1931).

References: VertNet: ${ }^{1}$ SBMNH, ${ }^{2}$ WFVZ; ${ }^{3}$ Lyle (1931).

\section{Order Passeriformes \\ Family Tyrannidae Genus Sayornis}

Sayornis phoebe (Latham, 1790) (TX) Eastern Phoebe

Localities: Campbell Co.: Panther Cave No. 1 (CM8)*; Claiborne Co.: Sour Kraut Cave (CB46)*, Buis Saltpeter Cave (CB48)*, Kings Saltpeter Cave (CB52)*; Knox Co.: 
Campbell Cave (KN1)*, Ebenezer Rising Cave (KN150)*; McMinn Co.: Small Cave (MM5)*, Too Small Cave (MM6)*; Monroe Co.: Lick Creek Cave (MO8)*, Alans Hideaway Cave (MO9)*; Rhea Co.: Marler Cave (RH4) ${ }^{1}$; Roane Co.: Berry Cave (RN3)*, Cave Creek Cave (RN5)*; Union Co.: Oaks Cave (UN5)*, Wright Cave (UN9)*, Big Cave (UN10)*, Rogers Hollow Cave (UN23)*, Ellison Hollow Cave (UN46)*.

Conservation status: IUCN: Least Concern; NatureServe: G5 (S5 in Tennessee).

Comments: This flycatcher is common throughout the eastern United States and is frequently encountered in entrances and the twilight zone of caves, where it nests on rocky ledges or in crevices.

References: ${ }^{1}$ Lewis (2005); * present study.

\section{Class Mammalia \\ Order Carnivora \\ Family Canidae \\ Genus Canis}

Canis latrans Say, 1823 (TX/AC) Coyote

Localities: McMinn Co.: Small Cave (MM5)*; Union Co.: Oaks Cave (UN5)*, Big Cave (UN10)*.

Conservation status: IUCN: Least Concern; NatureServe: G5 (S5 in Tennessee).

Comments: Fresh scat and tracks were observed just inside the entrance to Small Cave, and fresh scat was observed in the two Union County caves.

References: * present study.

\section{Family Procyonidae Genus Procyon}

Procyon lotor (Linnaeus, 1758) (TX) Raccoon

Localities: Blount Co.: Tory Shields Bluff Cave (BA56) ${ }^{1,2}$; Campbell Co.: Panther Cave No. 1 (CM8)*; Claiborne Co.: Sour Kraut Cave (CB46)*, Buis Saltpeter Cave (CB48)*, Kings Saltpeter Cave (CB52)*; Jefferson Co.: Tater Cave (JF8)*; Knox Co.: Campbell Cave (KN1)*, Mudflats Cave (KN9)*, Cruze Cave (KN24)*, Meads Quarry Cave (KN28)*, Unreported Cave (KN90)*, Chriscroft Cave (KN127)*; Loudon Co.: Phantom Insurgence Cave (LN22)*; McMinn Co.: Small Cave (MM5)*, Too Small Cave (MM6)*; Monroe Co.: Gay Cave (MO3)*, Morgan Cave (MO5)*, Nobletts Cave (MO6)*, Lick Creek Cave (MO8)*; Rhea Co.: Grassy Creek Cave $(\mathrm{RH} 2)^{*}$, Marler Cave $(\mathrm{RH} 4)^{3}$; Roane Co.: Berry Cave (RN3)*, Cave Creek Cave (RN5)*, Eblen Cave (RN6)*; Sevier Co.: Two County Cave (SV36)*; Union Co.: Oaks Cave (UN5)*, Wright Cave (UN9)*, Rogers Hollow Cave (UN23)*, Mossy Spring Cave (UN25)*.

Conservation status: IUCN: Least Concern; NatureServe: G5 (S5 in Tennessee).

Comments: Dead adults were found in Cruze Cave, Kings Saltpeter Cave, and Nobletts Cave, while tracks and/ or scat were observed in the other caves. Raccoons often enter caves in search of crayfish, frogs, salamanders, and other prey.
References: ${ }^{1,2}$ Wallace (1984, 2003); ${ }^{3}$ Lewis (2005); * present study.

\section{Order Chiroptera \\ Family Vespertilionidae \\ Genus Corynorhinus}

Corynorhinus rafinesquii (Lesson, 1827) (TX) Rafinesque's Big-Eared Bat

Localities: Blount Co.: Whiteoak Blowhole Cave $(\mathrm{BA} 2)^{11}$, Bull Cave $(\mathrm{BA} 3)^{1,8}$, Gregory Cave (BA4) $)^{1,6,7}$, Kelly Ridge Cave (BA6) ${ }^{1,8,10,11}$, Scott Gap Cave (BA7) $^{6,7,11}$, Calf Cave No. 2 (BA20) ${ }^{5}$; Greene Co.: Double Mouth Cave (GN6) ${ }^{1,9}$; Hancock Co.: unknown cave ${ }^{4}$; Hawkins Co.: Pearson Cave (HW12)ㄹ ${ }^{2}$, Monroe Co.: Ballplay Cave $(\mathrm{MO} 10)^{3}$; Sevier Co.: Stupkas Cave $(\mathrm{SV} 42)^{6,7}$.

Conservation status: IUCN: Least Concern; NatureServe: G3G4 (S3 in Tennessee).

Comments: Although we did not observe Rafinesque's Big-Eared Bats during our bioinventories, this species has been reported from several eastern Tennessee caves, primarily during winter hibernaculum surveys.

References: ${ }^{1}$ Bat Population Database; VertNet: ${ }^{2} \mathrm{KU}$, ${ }^{3} \mathrm{MVZ},{ }^{4} \mathrm{TNNH} ;{ }^{5}$ Rabinowitz and Nottingham (1979); ${ }^{6,7}$ Wallace (1984, 2003); ${ }^{8}$ Samoray (2011); ${ }^{9}$ Holliday (2012); ${ }^{10,11}$ Flock $(2013,2014)$.

\section{Genus Eptesicus}

Eptesicus fuscus (Beauvois, 1796) (TX) Big Brown Bat

Localities: Anderson Co.: Springhill Saltpeter Cave (AN3) ${ }^{9,10}$; Blount Co.: Whiteoak Blowhole Cave (BA2) ${ }^{4,5}$, Bull Cave (BA3) $)^{1,7}$, Gregory Cave (BA4) ${ }^{4,5}$, Kelly Ridge Cave (BA6) ${ }^{1,9,10}$, Whiteoak Saltpeter Cave (BA27) $)^{1,7,9,10}$; Campbell Co.: Meredith Cave (CM5) ${ }^{1,7}$, Norris Dam Cave $(\mathrm{CM} 7)^{1,8,9}$, Panther Cave No. 1 (CM8)*; Carter Co.: Carter Saltpeter Cave (CR1) $)^{1,6}$, Grindstaff Cave $(\mathrm{CR} 2)^{1,6,7,10}$, Renfro Cave $(\mathrm{CR} 6)^{10}$, Poga Road Cave $(\mathrm{CR} 31)^{1,6}$, Elk Mills Cave (CR34) ${ }^{1,6}$; Claiborne Co.: Sour Kraut Cave $(\mathrm{CB} 46)^{9}$, Buis Saltpeter Cave (CB48) ${ }^{9}$, cave near Harrogate ${ }^{3}$; Grainger Co.: Indian Cave (GA4) $)^{9,10, *}$; Greene Co.: Cochran Cave $(\mathrm{GN} 32)^{1,8}$; Hamblen Co.: Soard Cave (HB3) ${ }^{9}$, Corner Store Cave (HB22) ${ }^{10}$; Jefferson Co.: Tater Cave $(\mathrm{JF} 8)^{9}$, Rouse Cave $(\mathrm{JF} 26)^{9}$; Knox Co.: Campbell Cave (KN1)*, Mudflats Cave (KN9)*, Blowing Hole Cave (KN19) ${ }^{9}$, Meads Quarry Cave (KN28) ${ }^{1,8, *}$, Cave Spring Cave No. 2 (KN47) ${ }^{1,8}$, Kirkpatrick Cave (KN62)*; McMinn Co.: Small Cave (MM5)*; Meigs Co.: Eves Cave (ME2) ${ }^{10}$; Monroe Co.: Ballplay Cave (MO10) ${ }^{2}$; Roane Co.: Berry Cave (RN3)*, Cave Creek Cave $(\mathrm{RN} 5)^{10}$, Eblen Cave (RN6)*, Big Cave (RN13)*, Marble Bluff Cave $(\mathrm{RN} 19)^{1,10}$; Sullivan Co.: Morrell Cave (SL6) $^{1,6,7,9,10}$, Kaylor Cave (SL46) ${ }^{1,6}$; Union Co.: Oaks Cave (UN5) ${ }^{1,6-8}$, Herd O Coons Cave (UN32) ${ }^{9}$.

Conservation status: IUCN: Least Concern; NatureServe: G5 (S5 in Tennessee).

Journal of Cave and Karst Studies, April 2016•13 
Comments: Eptesicus fuscus is frequently encountered in caves, particularly in winter, where it often roosts alone or in small clusters on ledges or in crevices in cave walls.

References: ${ }^{1}$ Bat Population Database; VertNet: ${ }^{2}$ MVZ, ${ }^{3}$ OMNH; ${ }^{4,5}$ Wallace $(1984,2003) ;{ }^{6}$ Lamb and Wyckoff (2010); ${ }^{7}$ Samoray $(2011) ;{ }^{8}$ Holliday (2012); ${ }^{9,10}$ Flock $(2013,2014) ;{ }^{*}$ present study.

\section{Genus Lasiurus}

\section{Lasiurus borealis (Muller, 1776) (AC) Eastern Red Bat}

Localities: Rhea Co.: Grassy Creek Cave (RH2) ${ }^{1}$, Starve Rock Cave (RH7) ${ }^{1}$.

Conservation status: IUCN: Least Concern; NatureServe: G4 (S5 in Tennessee).

Comments: This bat is typically associated with forests but has been found in caves on occasion (Mohr, 1952; Quay and Miller, 1955; Myers, 1960). This species will swarm around cave entrances, particular in autumn; however, bats that enter caves typically die, presumably because they become disoriented and are unable to find their way back to an entrance (Best and Dusi, 2014).

References: ${ }^{1}$ VertNet: KU.

\section{Lasiurus cinereus (Beauvois, 1796) (TX/AC) Hoary Bat}

Localities: Campbell Co.: Meredith Cave (CM5) ${ }^{1}$; Rhea Co.: Starve Rock Cave (RH7) ${ }^{1}$.

Conservation status: IUCN: Least Concern; NatureServe: G4 (S5 in Tennessee).

Comments: This bat is typically associated with forests but has been documented roosting in caves (Myers, 1960; Best and Dusi, 2014).

References: ${ }^{1}$ VertNet: KU.

\section{Genus Myotis}

Myotis grisescens Howell, 1909 (TX) Gray Bat

Localities: Anderson Co.: Little Turtle Cave (AN38) ${ }^{5}$; Campbell Co.: Meredith Cave (CM5) ${ }^{9}$, Norris Dam Cave $(\mathrm{CM} 7)^{1,9}$, Panther Cave No. 1 (CM8) ${ }^{5}$; Claiborne Co.: English Cave $(\mathrm{CB} 9)^{1,12}$, Station Creek Cave $(\mathrm{CB} 17)^{9}$, Upper Coonsies Creek Cave (CB47) ${ }^{1,2,8,9}$; Cocke Co.: Rattling Cave $(\mathrm{CO} 2)^{1,8,9}$; Grainger Co.: Indian Cave (GA4) $)^{1,3,4,8,9,13,14, *}$, Coon Cave (GA12) ${ }^{9}$; Greene Co.: Arch Cave $(\mathrm{GN} 1)^{9}$, Cedar Creek Cave $(\mathrm{GN} 3)^{9}$, Stillhouse Cave (GN7) ${ }^{1,12}$, Cochran Cave $(\mathrm{GN} 32)^{1,9,13}$; Hancock Co.: Rockhouse Cave (HN27) ${ }^{9}$, Upstream Cave (HN77) ${ }^{1,12}$; Hawkins Co.: Hasson Cave (HW6) ${ }^{1}$, Horner Cave (HW9 $^{1,9}$, Pearson Cave $(H W 12)^{1,2,9,10,12-14}$; Jefferson Co.: Rouse Cave (JF26) ${ }^{13}$; Knox Co.: Mudflats Cave $(\mathrm{KN} 9)^{9}$, Baloney Cave $(\mathrm{KN} 18)^{9}$, Blowing Hole Cave $(\mathrm{KN} 19)^{9}$, Christian Cave (KN49)*; Loudon Co.: Ghost Cave (LN3) ${ }^{5}$, Browder Bluff Cave No. 3 (LN7) ${ }^{5}$, Phantom Insurgence Cave (LN22)*; Meigs Co.: Blythe Ferry Cave $(\mathrm{ME1})^{1,2,9}$, Eves Cave (ME2) ${ }^{1,9,13}$, Sensabaugh Cave (ME3) $^{1,2,9, *}$; Rhea Co.: Grassy Creek Cave (RH2) ${ }^{1,2,6-9, *}$, Starve Rock Cave (RH7), ${ }^{2,9}$ Roane Co.: Marble Bluff Cave (RN19) $^{1}$; Sullivan Co.: Morrell Cave (SL6) ${ }^{1,7-9,12-14}$; Union
Co.: Oaks Cave (UN5) $)^{1,2,8-12, *}$; Wright Cave (UN9) ${ }^{1,12}$, Lost Creek Cave (UN19) ${ }^{9}$.

Conservation status: IUCN: Near Threatened; NatureServe: G3 (S2 in Tennessee); listed as Endangered under the U.S. Endangered Species Act; listed as Endangered in Tennessee (Withers, 2009).

Comments: Myotis grisescens is one of three bats federally listed as endangered that can be found in Tennessee, the others being the Indiana Bat (M. sodalis) and the Northern Long-Eared Bat (M. septentrionalis). Populations throughout Tennessee are well-monitored because large colonies are only found in approximately eight caves nationally (Martin 2007). Indian Cave, Grassy Creek Cave, and Oaks Cave are sites for summer maternity colonies, while Sensabaugh Cave is a summer bachelor colony (Martin, 2007). Martin (2007) included Mudflats Cave and Blowing Hole Cave as Priority 4 sites, but the species has not been observed at these caves in recent years (this study). Gray Bats have not been reported previously from Christian Cave or Phantom Insurgence Cave. An estimated 250 bats and fresh guano piles were observed at Christian Cave on 17 September 2005. At Phantom Insurgence Cave on 30 August 2014, an estimated 2,000 bats were observed roosting above a pool in the large entrance chamber. Although no roosting bats were observed in Grassy Creek Cave at the time of the bioinventory, large and extensive guano piles from a recent summer colony and several skeletons of dead bats were present throughout the cave during our visit on 22 December 2014.

References: ${ }^{1}$ Bat Population Database; VertNet: ${ }^{2} \mathrm{KU}$, ${ }^{3}$ UMMZ, ${ }^{4}$ USNM; ${ }^{5}$ TNNH; ${ }^{6}$ Wallace (1989); ${ }^{7}$ Harvey (1994); ${ }^{8}$ Harvey and Britzke (2002); ${ }^{9}$ Martin (2007); ${ }^{10}$ Lamb and Wyckoff (2010); ${ }^{11}$ Samoray (2011); ${ }^{12}$ Holliday (2012); ${ }^{13,14}$ Flock $(2013,2014)$; * present study.

\section{Myotis leibii (Audubon \& Bachman, 1842) (TX) Eastern Small-Footed Bat}

Localities: Anderson Co.: Springhill Saltpeter Cave $(\mathrm{AN} 3)^{4}$; Blount Co.: Kelly Ridge Cave (BA6) ${ }^{1}$; Carter Co.: Poga Road Cave $(\mathrm{CR} 31)^{1,3}$, Elk Mills Cave $(\mathrm{CR} 34)^{1,3}$; Union Co.: Lost Creek Cave (UN19) ${ }^{2}$; Washington Co.: Hampton Cave ${ }^{2}$.

Conservation status: IUCN: Least Concern; NatureServe: G3G4 (S2S3 in Tennessee); listed as Deemed in Need of Management in Tennessee (Withers 2009).

Comments: The Eastern Small-Footed Bat is the smallest bat species in the eastern United States. It is rare throughout its range in Tennessee, where it is found primarily in the eastern two-thirds of the state. Many records of this species are from winter surveys, when it can be found hibernating in caves and mines. Hampton Cave is not known in the TCS database and could not be georeferenced.

References: ${ }^{1}$ Bat Population Database; VertNet: ${ }^{2}$ OMNH; ${ }^{3}$ Lamb and Wyckoff (2010); ${ }^{4}$ Flock (2013). 
Myotis lucifugus (LeConte, 1831) (TX) Little Brown Bat

Localities: Anderson Co.: Springhill Saltpeter Cave $(\mathrm{AN} 3)^{10,11}$, Toilet Bowl Cave (AN14) $)^{10}$; Blount Co.: Whiteoak Blowhole Cave (BA2) $)^{1,5-11}$, Bull Cave $(\text { BA3 })^{1,3,8}$, Gregory Cave (BA4) $)^{1,9-11}$, Kelly Ridge Cave (BA6) $^{1,8,10,11}$, Scott Gap Cave (BA7) ${ }^{1,8-11}$, Tuckaleechee Caverns (BA11)*, Rainbow Cave (BA26) ${ }^{1,8}$, Whiteoak Saltpeter Cave $(\mathrm{BA} 27)^{1,8-10}$, Tory Shields Bluff Cave $(B A 56)^{5,6}$; Campbell Co.: Norris Dam Cave $(\mathrm{CM} 7)^{10}$; Carter Co.: Grindstaff Cave (CR2) $)^{1,8}$; Claiborne Co.: English Cave (CB9) ${ }^{1,9}$, Sour Kraut Cave $(\mathrm{CB} 46)^{10}$, Buis Saltpeter Cave $(\mathrm{CB} 48)^{10}$; Grainger Co.: Indian Cave $(\mathrm{GA} 4)^{4}$; Hamblen Co.: Corner Store Cave (HB22) ${ }^{11}$; Knox Co.: Cruze Cave (KN24)*, Meads Quarry Cave (KN28)*; Meigs Co.: Eves Cave (ME2) ${ }^{10,11}$; Rhea Co.: Grassy Creek Cave (RH2) ${ }^{2}$; Roane Co.: Marble Bluff Cave (RN19) ${ }^{10}$; Sullivan Co.: Morrell Cave (SL6) ${ }^{1,8,11}$; Union Co.: Oaks Cave (UN5) ${ }^{2}$, Wright Cave (UN9) ${ }^{1,9}$, Jolley Saltpeter Cave $(\mathrm{UN} 12)^{11}$, Lost Creek Cave (UN19) ${ }^{1,2,9}$, Herd O Coons Cave (UN32) ${ }^{10}$.

Conservation status: IUCN: Least Concern; NatureServe: G3 (S5 in Tennessee).

Comments: The range of this species includes all of eastern Tennessee; however, only a few individuals were noted during this study. The individual observed in Tuckaleechee Caverns on 20 March 2014 had characteristic white fungal growth of white-nose syndrome on the muzzle. Myotis lucifugus populations throughout the eastern United States have sustained significant losses due to WNS, and Ingersoll et al. (2013) have suggested that its IUCN Red List status be increased from Least Concern to Endangered in some areas. Declines have also been noted for some eastern Tennessee populations (Flock 2013, 2014).

References: ${ }^{1}$ Bat Population Database; VertNet: ${ }^{2}$ KU, ${ }^{3}$ OMNH, ${ }^{4}$ UMMZ; ${ }^{5,6}$ Wallace $(1984,2003) ;{ }^{7}$ Lamb and Wyckoff (2010); ${ }^{8}$ Samoray (2011); ${ }^{9}$ Holliday (2012); ${ }^{10,11}$ Flock $(2013,2014)$; * present study.

\section{Myotis septentrionalis (Trovessart, 1897) (TX) Northern Long-Eared Bat}

Localities: Blount Co.: Whiteoak Blowhole Cave $(\mathrm{BA} 2)^{1,5-10}$, Bull Cave (BA3) ${ }^{1,3,8}$, Gregory Cave (BA4) $)^{1,9-}$ ${ }^{11}$, Kelly Ridge Cave (BA6) ${ }^{10}$, Scott Gap Cave (BA7) ${ }^{1,8}$, Whiteoak Saltpeter Cave (BA27) ${ }^{1,9,10}$; Carter Co.: Carter Saltpeter Cave $(\mathrm{CR} 1)^{1,7}$, Grindstaff Cave $(\mathrm{CR} 2)^{1,7}$; Claiborne Co.: Sour Kraut Cave $(\mathrm{CB} 46)^{10}$, Buis Saltpeter Cave $(\mathrm{CB} 48)^{10}$; Grainger Co.: Indian Cave $(\mathrm{GA} 4)^{4}$; Greene Co.: Cedar Creek Cave (GN3) ${ }^{4}$; Meigs Co.: Eves Cave (ME2) ${ }^{11}$; Rhea Co.: Grassy Creek Cave (RH2) ${ }^{2}$; Roane Co.: Cave Creek Cave $(\mathrm{RN} 5)^{11}$, Marble Bluff Cave (RN19) $)^{1,8,9,11}$; Sullivan Co.: Morrell Cave (SL6) ${ }^{1,7}$; Union Co.: Oaks Cave (UN5) $^{1,2,7, *}$, Wright Cave (UN9) ${ }^{1,9}$, Jolley Saltpeter Cave $(\mathrm{UN} 12)^{11}$, Herd O Coons Cave (UN32) ${ }^{10}$.
Conservation status: IUCN: Least Concern; NatureServe: G1G2 (S4 in Tennessee); listed as Threatened under the U.S. Endangered Species Act.

Comments: We observed a single individual at Oaks Cave in March 2015. United States Fish and Wildlife Service (2013) found that listing this species is warranted, as it is one of the bat species most impacted by white-nose syndrome, and proposed to list $M$. septentrionalis as an endangered species throughout its range. The species was listed as Threatened under the Endangered Species Act, effective 4 May 2015 (United States Fish and Wildlife Service, 2015).

References: ${ }^{1}$ Bat Population Database; VertNet: ${ }^{2} \mathrm{KU}$, ${ }^{3}$ OMNH, ${ }^{4}$ TNNH; ${ }^{5,6}$ Wallace $(1984,2003) ;{ }^{7}$ Lamb and Wyckoff (2010); ${ }^{8}$ Samoray (2011); ${ }^{9}$ Holliday (2012); ${ }^{10,11}$ Flock $(2013,2014)$; * present study.

\section{Myotis sodalis Miller \& Allen, 1928 (TX) Indiana Bat}

Localities: Blount Co.: Whiteoak Blowhole Cave (BA2) $)^{1,4-12}$, Bull Cave (BA3) $)^{1,2,4,5,7,9}$, Kelly Ridge Cave (BA6) $^{1,7,9,11,12}$, Scott Gap Cave (BA7) ${ }^{1,4,5,7,9-12}$, Rainbow Cave (BA26) ${ }^{1,9}$, Whiteoak Saltpeter Cave (BA27) ${ }^{1,9,11,12}$; Campbell Co.: Meredith Cave (CM5) ${ }^{7}$, Norris Dam Cave $(\mathrm{CM} 7)^{7}$; Claiborne Co.: English Cave (CB9 $)^{3}$; Grainger Co.: Indian Cave (GA4) ${ }^{7}$, Coon Cave $(\mathrm{GA} 12)^{3}$; Hawkins Co.: Pearson Cave (HW12) ${ }^{1,7}$; Union Co.: Jolley Saltpeter Cave (UN12).

Conservation status: IUCN: Endangered A2ac; NatureServe: G2 (S1 in Tennessee); listed as Endangered under the U.S. Endangered Species Act; listed as Endangered in Tennessee (Withers, 2009).

Comments: We did not observe any Indiana Bats during our bioinventories. However, several priority sites exist in eastern Tennessee for this endangered species (United States Fish and Wildlife Service, 2007).

References: ${ }^{1}$ Bat Population Database; VertNet: ${ }^{2}$ OMNH, ${ }^{3}$ TNNH; ${ }^{4,5}$ Wallace $(1984,2003) ;{ }^{6}$ Harvey and Britzke (2002); ${ }^{7}$ United States Fish and Wildlife Service (2007); ${ }^{8}$ Lamb and Wyckoff (2010); ${ }^{9}$ Samoray (2011); ${ }^{10}$ Holliday (2012); ${ }^{11,12}$ Flock (2013, 2014).

\section{Genus Perimyotis}

Perimyotis subflavus (Cuvier, 1832) (TX) Tri-Colored Bat

Localities: Anderson Co.: Springhill Saltpeter Cave $(\mathrm{AN} 3)^{13,14}$, Toilet Bowel Cave (AN14) $)^{13}$; Blount Co.: Whiteoak Blowhole Cave (BA2) ${ }^{1,6-8,10-14}$ Bull Cave (BA3) ${ }^{1,4,6-8,11}$, Gregory Cave (BA4) ${ }^{1,6-8,12-14}$, Kelly Ridge Cave (BA6) $)^{1,11,13,14}$, Scott Gap Cave (BA7) $^{1,6-8,11-14}$, Tuckaleechee Caverns (BA11)*, Hatcher Cave (BA12) ${ }^{6}$, Calf Cave No. 1 (BA19) ${ }^{7}$, Calf Cave No. 2 (BA20) $)^{7,8}$, Rainbow Cave (BA26) ${ }^{1,7,8,11}$, Whiteoak Saltpeter Cave (BA27) ${ }^{1,6,11-14}$, Tory Shields Bluff Cave (BA56) ${ }^{6-}$ ${ }^{8}$; Campbell Co.: Meredith Cave (CM5) ${ }^{1,11}$, Norris Dam Cave (CM7) ${ }^{1,10-13}$, Panther Cave No. 1 (CM8)*; Carter Co.: Carter Saltpeter Cave $(\mathrm{CR} 1)^{1,10, *}$, Grindstaff Cave $(\mathrm{CR} 2)^{1,10-12,14}$, Renfro Cave (CR6) ${ }^{14}$, Poga Road Cave $(\mathrm{CR} 31)^{1,10}$, Elk Mills Cave (CR34) ${ }^{1,10}$; Sculpture Cave 
$(\text { CR63) })^{1,10}$, Conway Cave $\left(\right.$ CR66) ${ }^{1,10}$; Claiborne Co.: English Cave (CB9) ${ }^{1,12}$, Sour Kraut Cave (CB46) ${ }^{13}$, Buis Saltpeter Cave $(\mathrm{CB} 48)^{1,11,13}$; Grainger Co.: Indian Cave $(\mathrm{GA} 4)^{13,14, *}$; Greene Co.: Poplar Cave $(\mathrm{GN} 5)^{1,12}$, Still House Cave (GN7) ${ }^{1,12}$, Cochran Cave $(\mathrm{GN} 32)^{1,12,13}$, Afton Cave (GN69) ${ }^{13}$; Hamblen Co.: Soard Cave $(\mathrm{HB} 3)^{13}$, Saltpeter Cave $(\mathrm{HB} 4)^{14}$, Corner Store Cave $(\mathrm{HB} 22)^{14}$; Hancock Co.: Cantwell Valley Cave (HN2) ${ }^{1,12}$, Upstream Cave (HN77) ${ }^{1,12}$; Jefferson Co.: Tater Cave (JF8) ${ }^{13, *}$, Rouse Cave (JF26) ${ }^{13}$; Knox Co.: Campbell Cave (KN1)*, Cherokee Bluff Cave (KN4)*, Mudflats Cave (KN9)*, Carter Cave (KN14)*, Keller Bend Cave (KN16)*, Blowing Hole Cave $(\mathrm{KN} 19)^{13, *}$, Cherokee Caverns (KN22)*, Cruze Cave (KN24)*, Meads Quarry Cave (KN28) ${ }^{1,12, *}$, Cave Spring Cave No. 2 (KN47) ${ }^{1,12}$, Christian Cave (KN49) ${ }^{1,12, *}$, Keller Bluff Cave No. 1 (KN61) Kirkpatrick Cave (KN62)*, Unreported Cave (KN90)*, Brents Cave (KN112)*, Chriscroft Cave (KN127)*, The Lost Puddle (KN145)*, Meads River Cave (KN151)*; Loudon Co.: Blankenship Cave (LN1) ${ }^{14, *}$; McMinn Co.: Small Cave (MM5)*; Meigs Co.: Blythe Ferry Cave $(\mathrm{ME} 1)^{14}$, Eves Cave (ME2) ${ }^{13,14}$; Monroe Co.: Gay Cave (MO3)*, Morgan Cave (MO5)*, Nobletts Cave (MO6)*, Lick Creek Cave (MO8)*, Alans Hideaway Cave (MO9)*, Ballplay Cave (MO10) ${ }^{3}$, Luther Cave (MO11) ${ }^{13}$; Polk Co.: Gee Cave (PO1) ${ }^{1,12-14}$; Rhea Co.: Grassy Creek Cave $(\mathrm{RH} 2)^{2}, *$, Marler Cave (RH4) ${ }^{9}$; Roane Co.: Berry Cave (RN3)*, Cave Creek Cave (RN5) ${ }^{14, *}$, Eblen Cave (RN6)*, Big Cave (RN13)*, Chimney Cave (RN14)*, Marble Bluff Cave (RN19) $)^{1,10-14}$, Smith Cave $(\mathrm{RN} 37)^{1,12}$; Sevier Co.: Stupkas Cave (SV42) $)^{7,8}$; Sullivan Co.: Morrell Cave (SL6) $^{1,10-14}$, Kaylor Cave (SL46) ${ }^{1,10}$; Union Co.: Oaks Cave (UN5) ${ }^{1,10-12, *}$, Wright Cave (UN9) ${ }^{1,12, *}$, Jolley Saltpeter Cave $(\mathrm{UN} 12)^{14}$, Lost Creek Cave (UN19) ${ }^{1,12}$, Rogers Hollow Cave (UN23)*, Herd O Coons Cave (UN32) $^{13}$; Washington Co.: The Man Cave (WS8) ${ }^{14}$, Hampton Cave ${ }^{4}$.

Conservation status: IUCN: Least Concern; NatureServe: G3 (S5 in Tennessee).

Comments: Perimyotis subflavus is among the most commonly encountered bats in caves of eastern Tennessee, and this bat was the most prevalent in our study. Seven dead and three live $P$. subflavus with characteristic whitenose syndrome symptoms were observed at Eblen Cave in May 2013 (Fig. 2). Three Tri-Colored Bats with characteristic WNS symptoms were observed at both Wright Cave and Oaks Cave in Union County in March 2015. TriColored Bat populations are suffering significant losses due to WNS throughout its range (Turner et al., 2011; Ingersoll et al., 2013). The strongest effects of the disease have been on populations in localities with more severe winter climate, where hibernation time is longer and the fungal pathogen has greater opportunity to infect hibernating bats. Reexamination of the IUCN Red List status for this species in the coming years may be needed (Ingersoll et al.,
2013). Hampton Cave is not known in the TCS database and could not be georeferenced.

References: ${ }^{1}$ Bat Population Database; VertNet: ${ }^{2} \mathrm{KU}$, ${ }^{3}$ MVZ, ${ }^{4}$ OMNH, ${ }^{5}$ TNNH; ${ }^{6}$ Rabinowitz (1981); ${ }^{7,8}$ Wallace $(1984,2003) ;{ }^{9}$ Lewis (2005); ${ }^{10}$ Lamb and Wyckoff (2010); ${ }^{11}$ Samoray (2011); ${ }^{12}$ Holliday (2012); ${ }^{13,14}$ Flock (2013, 2014); * present study.

\section{Order Rodentia \\ Family Castoridae \\ Genus Castor}

Castor canadensis Kuhl, 1820 (TX) American Beaver

Localities: Knox Co.: Ebenezer Rising Cave (KN150)*; Roane Co.: Eblen Cave (RN6)*.

Conservation status: IUCN: Least Concern; NatureServe: G5 (S5 in Tennessee).

Comments: A beaver and associated lodge were observed just inside the entrance of Ebenezer Rising Cave. Two adults were observed near the cave stream within Eblen Cave on 30 December 2005.

References: * present study.

\section{Family Cricetidae \\ Genus Neotoma}

Neotoma magister Baird, 1858 (TX) Allegheny Woodrat

Localities: Carter Co.: Grindstaff Cave (CR2) ${ }^{2}$; Hancock Co.: Little Rockhouse Cave (HN19) ${ }^{1}$, unknown cave $^{1}$; Hawkins Co.: Barretts Cave (HW26) ${ }^{1}$; Knox Co.: Carter Cave (KN14)*, Keller Bend Cave (KN16)*, Blowing Hole Cave (KN19)*, Ebenezer Rising Cave (KN150)*; Monroe Co.: Gay Cave (MO3)*, Morgan Cave (MO5)*; Rhea Co.: Marler Cave (RH4) ${ }^{3}$; Union Co.: Mossy Spring Cave (UN25)*; Washington Co.: Keplinger Cave (WS3) ${ }^{1}$.

Conservation status: IUCN: Near Threatened; NatureServe: G3G4 (S3 in Tennessee); listed as Deemed in Need of Management in Tennessee (Withers 2009).

Comments: Although this species was not observed directly, evidence for its occurrence (i.e., latrines, caches, and nests) was noted in several caves. Kennedy et al. (2012) state that $N$. magister occurs only in central Tennessee, with $N$. floridana replacing this species west of the Tennessee River in western Tennessee and also in the Appalachian Valley and Ridge of eastern Tennessee. But Best and Dusi (2014) show the range of $N$. magister includes eastern Tennessee. These two species are identical in general appearance and can be distinguished by the shape of the palate and by genetic examination.

References: VertNet: ${ }^{1}$ TNNH; ${ }^{2}$ Conaway and Howell (1953); ${ }^{3}$ Lewis (2005); * present study.

\section{Genus Peromyscus \\ Peromyscus gossypinus (LeConte, 1853) (TX) Cotton Deermouse \\ Localities: Grainger Co.: Indian Cave (GA4) ${ }^{1}$. \\ Conservation status: IUCN: Least Concern; Nature- Serve: G5 (S5 in Tennessee).}




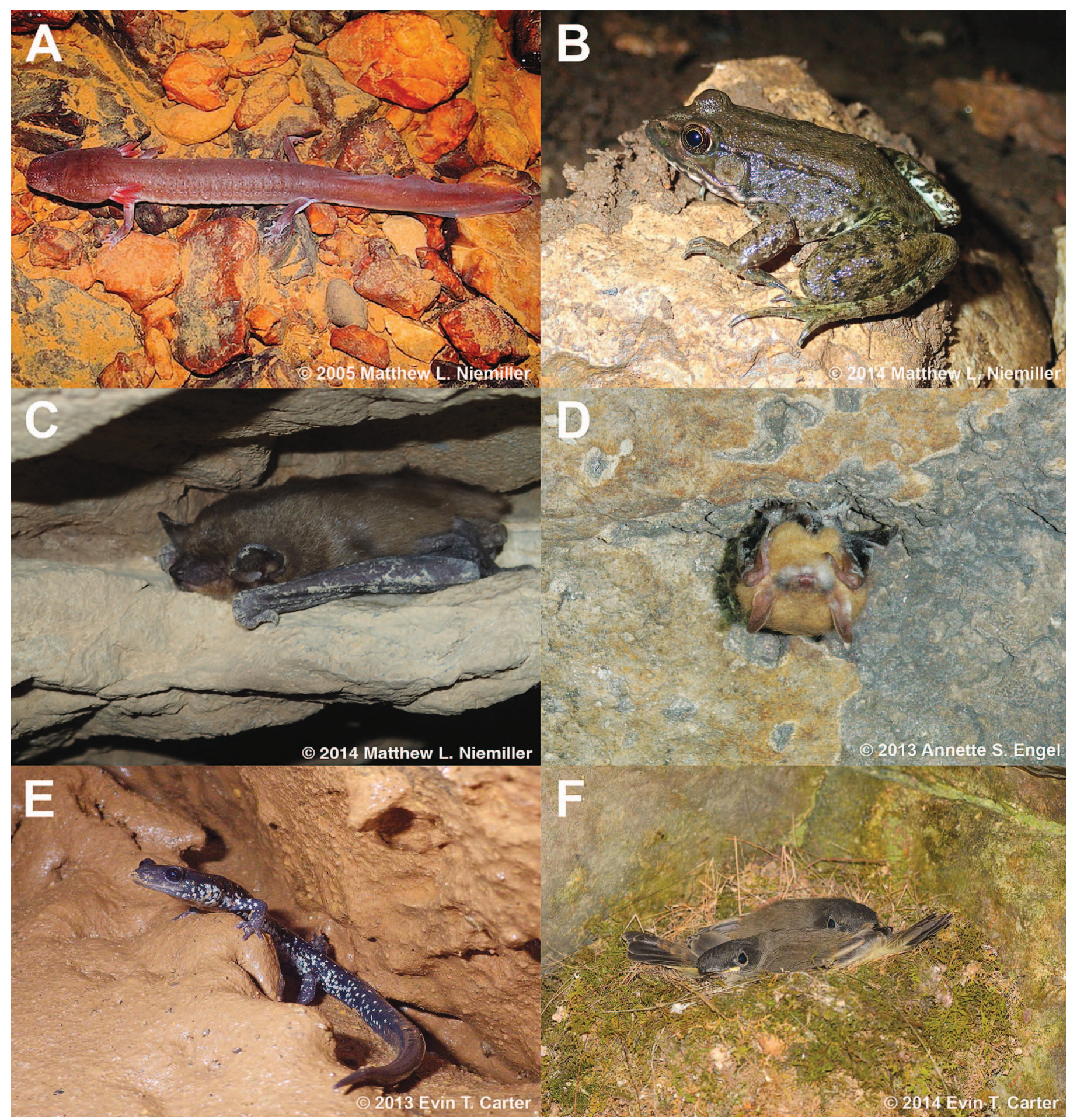

Figure 2. Vertebrate cave life documented in Appalachian Valley and Ridge and Blue Ridge Mountains caves of eastern Tennessee: A) an adult Berry Cave Salamander Gyrinophilus gulolineatus from Roane County; B) Green Frog Lithobates clamitans from Loudon County; C) Big Brown Bat Eptesicus fuscus from Knox County; D) Tri-Colored Bat Perimyotis subflavus from Roane County exhibiting fungal growth characteristic of white-nose syndrome; E) Northern Slimy Salamander Plethodon glutinosus from Monroe County; and F) fledgling Eastern Phoebes Sayornis phoebe from McMinn County. 
Comments: Two specimens were collected from Indian Cave in December 1911.

References: VertNet: ${ }^{1}$ UMMZ.

\section{Peromyscus leucopus (Rafinesque, 1818) (TX) White-Footed Deermouse}

Localities: Campbell Co.: Panther Cave No. 1 (CM8)*; Meigs Co.: Sensabaugh Cave (ME3)*.

Conservation status: IUCN: Least Concern; NatureServe: G5 (S5 in Tennessee).

Comments: Two adults and a nest were observed just inside the entrance to Panther Cave No. 1. Three adults were observed in the dark zone of the main upper-level passage at Sensabaugh Cave on 31 August 2014.

References: * present study.

\section{Peromyscus sp. (TX/AC) Unidentified mouse}

Localities: Knox Co.: Campbell Cave (KN1)*; Union Co.: Big Cave (UN10)*.

Conservation status: Not applicable.

Comments: Mouse scat was found in the dark zone on a mud bank at Big Cave.

References: * present study.

\section{Order Soricomorpha Family Soricidae Genus Blarina Blarina brevicauda (Say, 1823) (AC) Northern Short-Tailed Shrew}

Localities: Knox Co.: Meads Quarry Cave (KN28)*.

Conservation status: IUCN: Least Concern; NatureServe: G5 (S5 in Tennessee).

Comments: A recently deceased $B$. brevicauda was found in a crawl passage in Meads Quarry Cave between the downstream and main entrances.

References: * present study.

Summary of Bioinventory and Literature Records

For this study, we bioinventoried 56 caves during 107 individual surveys (Table 1) and documented 38 vertebrate taxa that represented 275 total species occurrence records. A mean of $4.9 \pm 2.9$ taxa were observed per cave. Ten or more species were documented from four caves: Meads Quarry Cave (KN28; 14 species), Berry Cave (RN3; 12 species), Small Cave (MM5; 11 species), and Meads River Cave (KN151; 10 species). We were able to verify 36 vertebrate species: 6 fishes, 15 amphibians (5 anurans and 10 salamanders), 3 reptiles, 1 bird, and 11 mammals. Amphibians accounted for most vertebrate occurrences (47.3\% of the total records), with salamanders comprising the most prevalent order of vertebrates $(37.8 \%$ of the total records). Bats (20.7\% of the total records) and non-bat mammals $(16.0 \%$ of the total records) also were frequently documented during bioinventories. No stygobiont fishes were documented during bioinventories, although three cyprinid, two centrarchid, one cottid, and one ictalurid species were recorded. The Cave Salamander Eurycea lucifuga, Tri-Colored Bat Perimyotis subflavus, Raccoon Procyon lotor, Northern Slimy Salamander Plethodon glutinosus, and Spring Salamander Gyrinophilus porphyriticus were the five most commonly encountered species, found in 38, 37, 27, 19, and 18 caves, respectively (Table 2). Thirteen observed taxa were considered accidentals in cave habitats, 14 were trogloxenes, 5 were troglophiles, and 1 was a stygobite (Table 2). All taxa classified as accidentals are considered common in surface habitats. The ecological classification of five taxa (Ameiurus natalis, Eurycea longicauda, Plethodon dorsalis, Canis latrans, and Peromyscus sp.) could not be reliably assigned to one category, and therefore three taxa were considered accidentals or either trogloxenes or stygoxenes and two were considered troglophiles or trogloxenes.

The amount of available habitat may be positively related to species richness of troglobionts in caves at the regional scale, where areas of higher cave density (as a proxy for available habitat) harbor greater species richness (Christman and Culver, 2001; Culver et al., 2003, 2006), or at the scale of individual caves, where larger caves support greater species richness (Schneider and Culver, 2004). From our data, there was a positive correlation between vertebrate species richness and cave length as a proxy for available habitat after accounting for sampling effort, according to weighted Pearson correlation analysis (correlation coefficient $r=0.340, P$-value $=0.01$ ). However, interpreting such a trend is likely confounded by variation in cave length and complexity, availability of habitat types such as wet or dry, surface land cover above caves and around entrances, and seasonal timing of cave bioinventories. In particular, seasonality might be especially important, as certain species, such as hibernating bats, are much more likely to be observed in caves during particular seasons. A positive correlation existed between species richness and the number of bioinventories conducted at a cave $(r=0.498, P$-value $<0.001)$. Consequently, multiple bioinventories spanning different seasons are recommended to adequately document the vertebrate fauna of individual caves. The presence of substantial aquatic habitat also appears important. Wet caves $(5.8 \pm 3.2$ taxa) exhibited greater species richness than dry caves $(3.3 \pm 1.3$ taxa), according to Wilcoxon rank sum test value $W=3008, P$-value $<0.001$. Unsurprisingly, predominantly aquatic caves contained species most often associated with aquatic habitats, and mammals were most often observed in dry caves.

Our review of available literature, biodiversity databases, and museum collections yielded an additional 298 species occurrences, 258 of which were unique from species occurrences recorded during our bioinventories, and 40 that were the same as species occurrences generated through our bioinventories. Bats accounted for the vast majority of additional occurrences (65.1\%), with many of these occurrences documented during winter white-nose syndrome surveys in the last five years (e.g., Lamb and 
Wyckoff, 2010; Samoray, 2011; Holliday, 2012; Flock, 2013, 2014). From the literature, 10 or more vertebrate species were documented from 11 caves: Gregory Cave (BA4; 15 species), Meads Quarry Cave (KN28; 14 species), Small Cave (MM5; 12 species), Berry Cave (RN3; 12 species), Whiteoak Blowhole Cave (BA2; 11 species), Indian Cave (GA4; 11 species), Cave Creek Cave (RN5; 11 species), Panther Cave No. 1 (CM8; 10 species), Mudflats Cave (KN9; 10 species), Meads River Cave (KN151; 10 species), and Grassy Creek Cave (RH2; 10 species).

Of the 533 unique species occurrence records, 522 could be georeferenced to 132 caves, including the 56 caves bioinventoried in this study. In total, 54 vertebrate taxa have been documented in eastern Tennessee caves (Table 2), including 8 fishes, 19 amphibians (8 anurans and 11 salamanders), 6 reptiles (5 snakes and 1 turtle), 3 birds, and 18 mammals (10 bats and 8 non-bat mammals). The Tri-Colored Bat (Perimyotis subflavus), Cave Salamander (Eurycea lucifuga), Big Brown Bat (Eptesicus fuscus), Gray Bat (Myotis grisescens), and Northern Slimy Salamander (Plethodon glutinosus) were the five most commonly encountered species, with 89,48 , 41, 37, and 31 total locations, respectively (Table 2). Twenty-one taxa were considered accidentals in cave habitats, 18 were trogloxenes, 5 were troglophiles, and 1 was a stygobite (Table 2). All taxa classified as accidentals are considered common in surface habitats. The ecological classification of nine taxa (Ameiurus natalis, Lithobates sylvaticus, Eurycea longicauda, Plethodon dorsalis, Cathartes aura, Coragyps atratus, Lasiurus cinereus, Canis latrans, and Peromyscus sp.) could not reliably be assigned to one category, and therefore seven taxa were considered accidentals or either trogloxenes or stygoxenes, and two were considered troglophiles or trogloxenes.

\section{Species of Conservation Concern}

Thirty-two of the vertebrate taxa in the Appalachian Valley and Ridge and The Blue Ridge Mountains karst areas of eastern Tennessee rank as Least Concern under IUCN Red List criteria (Table 2). However, two taxa rank as Near Threatened, one as Vulnerable, and one as Endangered. Based on NatureServe conservation rank criteria, thirty of the species are globally ranked as Secure (G5), one as Apparently Secure or Vulnerable (G3G4), three as Vulnerable (G3), one as Imperiled or Vulnerable (G2G3), and one as Critically Imperiled (G1). At the state level, NatureServe conservation ranks are similar to the IUCN listings, with thirty species Secure (S5), three Apparently Secure (S4), one Vulnerable (S3), one Imperiled (S2), and one Critically Imperiled (S1). Three of the observed species are tracked by the state of Tennessee (Withers, 2009). Myotis grisescens (Gray Bat) is listed as Endangered, Gyrinophilus gulolineatus (Berry Cave Salamander) as Threatened, and Neotoma magister (Allegheny
Woodrat) is Deemed in Need of Management. All of the accidental species observed in bioinventoried caves have a NatureServe global rank of G5 (Secure), with the exception of Lasiurus borealis, which has a global rank of G4 (Apparently Secure).

The Berry Cave Salamander (Gyrinophilus gulolineatus) is the only stygobiontic vertebrate known from the Valley and Ridge in Tennessee (Brandon, 1965, 1966; Miller and Niemiller, 2007, 2008; Niemiller et al., 2010). This species is a rare neotenic salamander previously known from just nine localities in Knox, McMinn, Meigs, and Roane counties (Miller and Niemiller, 2008; Niemiller and Miller, 2010, 2011). We increased the known localities of this rare salamander within that area to 11 caves. Because of few occurrences, low population densities, and threats associated with urban development and quarrying activities, $G$. gulolineatus is a candidate species for listing on the U.S. Endangered Species List (U.S. Fish and Wildlife Service Species Assessment; http://ecos.fws.gov/docs/candidate/ assessments/2014/r4/D03B_V01.pdf).

We documented five bat species from 39 caves. Four species are considered Vulnerable by NatureServe, and two species, M. grisescens (Gray Bat) and M. septentrionalis (Northern Long-Eared Bat), are listed on the U.S. Endangered Species List as Endangered and Threatened, respectively. Five caves housed populations of $M$. grisescens that were either directly observed or hypothesized to occur on the basis of recent evidence of inhabitation such as fresh guano piles. All of these $M$. grisescens occurrences, except Loudon County, had been previously reported; see the range map compiled by Tennessee Bat Working Group. Myotis septentrionalis was recently listed as Threatened under the Endangered Species Act in May 2015 (United States Fish and Wildlife Service, 2015) because of population declines associated with the emergent infectious disease white-nose syndrome, which is caused by the fungus Pseudogymnoascus destructans (Pseudeurotiaceae). Although we only observed a single individual, at Oaks Cave in Union County in March 2015, M. septentrionalis is known from 20 additional caves in eastern Tennessee (Table 2). The distribution of $M$. sodalis (Indiana Bat) extends into eastern Tennessee with both hibernacula and summer non-maternity roosting colonies; it is also a federally endangered species. This bat was not detected in any of the bioinventoried caves, but is known from 13 caves in eastern Tennessee. The state of Tennessee tracks $M$. leibii (Eastern Small-Footed Bat), because it is Deemed in Need of Management. We did not observe this species, but it has been reported from six caves in eastern Tennessee.

The bats M. lucifugus (Little Brown Bat) and Perimyotis subflavus (Tri-Colored Bat) were previously considered Secure (G5), but their respective NatureServe conservation ranks were downgraded in 2012 to at best Vulnerable (G3) because of severe population declines. These declines were 
Table 3. Number of georeferenced vertebrate records and taxa from the bioinventory reported in this paper and from the literature for counties in the kast area of the Appalachian Valley and Ridge and adjacent Blue Ridge Mountains. The number of caves with records is compared to the number of caves known to the Tennessee Cave Survey.

\begin{tabular}{|c|c|c|c|c|c|}
\hline County & Number Records & Number Taxa & Sampled Caves & Total AVR-BRM Caves ${ }^{\mathrm{a}}$ & Percent \\
\hline Anderson & 8 & 6 & 4 & 66 & 6.1 \\
\hline Blount & 71 & 23 & 12 & 87 & 13.8 \\
\hline Bradley & 0 & 0 & 0 & 3 & 0.0 \\
\hline Campbell & 20 & 13 & 3 & 38 & 7.9 \\
\hline Carter & 20 & 8 & 8 & 70 & 11.4 \\
\hline Claiborne & 24 & 10 & 6 & 154 & 3.9 \\
\hline Cocke & 1 & 1 & 1 & 63 & 1.6 \\
\hline Grainger & 13 & 11 & 2 & 106 & 1.9 \\
\hline Greene & 11 & 5 & 7 & 112 & 6.3 \\
\hline Hamblen & 6 & 3 & 3 & 81 & 3.7 \\
\hline Hamilton & 0 & 0 & 0 & 35 & 0.0 \\
\hline Hancock & 6 & 4 & 5 & 81 & 6.2 \\
\hline Hawkins & 8 & 5 & 5 & 69 & 7.2 \\
\hline Jefferson & 13 & 8 & 4 & 104 & 3.8 \\
\hline Johnson & 0 & 0 & 0 & 25 & 0.0 \\
\hline Knox & 114 & 29 & 24 & 165 & 14.5 \\
\hline Loudon & 15 & 11 & 5 & 22 & 22.7 \\
\hline McMinn & 18 & 12 & 3 & 12 & 25.0 \\
\hline Meigs & 13 & 10 & 3 & 4 & 75.0 \\
\hline Monroe & 31 & 15 & 9 & 13 & 69.2 \\
\hline Polk & 1 & 1 & 1 & 6 & 16.7 \\
\hline Rhea & 20 & 15 & 3 & 8 & 37.5 \\
\hline Roane & 42 & 18 & 7 & 50 & 14.0 \\
\hline Sevier & 8 & 8 & 2 & 66 & 3.0 \\
\hline Sullivan & 9 & 7 & 3 & 175 & 1.7 \\
\hline Unicoi & 0 & 0 & 0 & 9 & 0.0 \\
\hline Union & 47 & 16 & 10 & 56 & 17.9 \\
\hline Washington & 3 & 3 & 2 & 42 & 4.8 \\
\hline Total & 522 & $54^{\mathrm{b}}$ & 132 & 1722 & 7.7 \\
\hline
\end{tabular}

${ }^{\mathrm{a}} \mathrm{AVR}=$ Appalachian Valley and Ridge; BRM $=$ Blue Ridge Mountains.

${ }^{\mathrm{b}}$ Number of distinct taxa.

attributed to high mortality from white-nose syndrome (Frick et al., 2010; Maher et al., 2012; Verant et al., 2012; Minnis and Lindner, 2013). The first confirmed cases of bats infected with white-nose syndrome in Tennessee were in winter 2009-2010 (Lamb and Wyckoff, 2010). Although thought to be widely distributed across eastern Tennessee, $M$. lucifugus was observed in just three caves during this study. At Tuckaleechee Caverns in Blount County, one specimen had the characteristic white fungal growth on the muzzle on 20 March 2014. The most frequently encountered bat species was $P$. subflavus, which we observed in 37 of 56 caves $(66.1 \%)$ surveyed. Evidence of white-nose syndrome infecting $P$. subflavus was observed at Eblen Cave in Roane County on 15 May 2013. In this cave, a single bat was discovered with white fungal growth on the muzzle, and several dead bats were observed throughout the cave. Evidence of white-nose syndrome infecting $P$. subflavus was also observed in March 2015 at Oaks Cave and Wright Cave in Union County. The disease was first detected in Blount County in 2012 (Holliday, 2012), Roane County in 2014 (Flock, 2014), and Union County in 2013 (Flock, 2013).

\section{Conclusions and Future Directions}

Caves and associated subterranean habitats are important for many vertebrate species for reproduction, hibernation, refuge, or other aspects of their life histories. Although caves have long been recognized as critical habitats for several species of bats, the importance of caves for other vertebrate taxa has received less attention, with the exception of some salamanders (e.g., Niemiller and Miller, 2009; Goricki et al., 2012). This study advances our understanding of cave use by vertebrates by providing insights into the richness and distribution of vertebrate taxa in the Appalachian Valley and Ridge and the Blue 
Ridge Mountains of eastern Tennessee. Prior to this study, 298 vertebrate occurrence records representing 35 taxa existed from at least 100 caves that could be georeferenced. Our efforts nearly doubled the number of records by generating an additional 235 unique species occurrence records for 36 taxa from 56 caves. Thirty-two of the 56 caves previously lacked any vertebrate records. In combination with literature data, 54 vertebrate taxa in total have been documented from 132 caves that could be georeferenced in 24 counties in eastern Tennessee. Significant numbers of fishes, amphibians, reptiles, birds, and mammals use caves in eastern Tennessee, and several species regularly use caves. However, overall only $7.7 \%$ of known caves in those parts of eastern Tennessee now have vertebrate occurrence records. Few caves in eastern Tennessee have had comprehensive bioinventories, and only a small fraction of those have had repeated bioinventories for all vertebrates, with the exception of periodic surveys of winter hibernating bats at select caves (e.g., Lamb and Wyckoff, 2010; Samoray, 2011; Holliday, 2012; Flock, 2013, 2014). Moreover, several karst areas still remain poorly investigated, particularly in Bradley, Claiborne, Cocke, Grainger, Hamblen, Hamilton, Jefferson, Johnson, Sevier, Sullivan, and Unicoi counties, where less than $5 \%$ of known caves in each county have a vertebrate occurrence record (Table 3). A growing body of literature suggests that caves provide important habitat for nontroglobiontic vertebrates. Caves should continue to be considered as resources to be protected in the management and conservation of vertebrate fauna.

\section{ACKNOWLEDGEMENTS}

Funding for this study was provided by the Cave Conservancy Foundation, Tennessee Wildlife Resources Agency, Illinois Natural History Survey, Yale Institute for Biospheric Studies, University of Tennessee, and Middle Tennessee State University. Bioinventories were conducted in accordance with protocols approved by the Institutional Animal Care and use Committee at Middle Tennessee State University, the University of Tennessee-Knoxville, and Yale University, and under authorization of the Tennessee Wildlife Resources Agency (permit nos. 1585 and 1605). We thank the following individuals for assistance arranging access to caves and with bioinventories: P. Beute, J. Bowers, K. Brannen, T. Brown, G. Capshaw, J.S. Carter, P.B. Colclough, R. Dave, N. Davidai, D. Dittrich-Reed, S. Duncan, S. Engel, A. England, L. Faust, M. Finkle, B.M. Fitzpatrick, R. Fitzpatrick, D. Fong, B.M. Glorioso, K. Hanksnecht, T. Haspel-Soares, L.E. Hayter, M. Healy, S. Hendrickson, P. Hudson, M. Jackson, S. Jones, S.W. Keenan, C. Kerr, R. Lawton, T. Loring, N. Mann, J. Manneschmidt, Z. Marion, A. McAnally, J.P. McClendon, D.J. McCubbins, B.T. Miller, A. Moni, G. Moni, B. Moxley, C. Ogle, M. Ogle, M.S. Osbourn, D. Pelren, M.L. Porter, M. Post, R.
Quasney, R.G. Reynolds, P. Shah, M. Simmons, M.E. Slay, D. Soares, M. Todd-Thompson, M. Tomlinson, J. Turnbough, N. Turnbough, R. Van Fleet, J. Welch, S. Young, and E. Yost. The Tennessee Natural Heritage Program provided data regarding vertebrate occurrences in caves of Tennessee. We thank C. Holliday (The Nature Conservancy), G. Moni (Tennessee Cave Survey), and C. Sutherland (Tennessee Cave Survey) for assistance with georeferencing caves.

\section{REFERENCES}

Armstrong, J.G., and Williams, J.C., 1971, Cave and spring fishes of the southern bend of the Tennessee River: Journal of the Tennessee Academy of Science, v. 46, p. 107-115.

Bailey, V., 1933, Cave life of Kentucky, mainly in the Mammoth Cave region: American Midland Naturalist, v. 14, p. 385-634.

Bailey, S., 1988, Grapevine Cave: Der Fledermaus, v. 16, no. 3 (reprinted, Speleo Digest 1988, p. 212).

Barr, T.C., Jr., 1968, Cave ecology and the evolution of troglobites, in Dobzhansky, T., Hecht, M.K., and Steere, W.C., eds., Evolutionary Biology, vol. 2: New York, Appleton-Century-crofts, p. $35-102$.

Barr, T.C., Jr., 1953, Notes on the occurrence of ranid frogs in caves: Copeia, v. 1953, p. 60-61.

Best, T.L., and Dusi, J.L., 2014, Mammals of Alabama: Tuscaloosa, Alabama, The University of Alabama Press, $496 \mathrm{p}$.

Black, J., 1971, The cave life of Oklahoma. A preliminary study (excluding Chiroptera): Oklahoma Underground, v. 4, p. 2-53.

Brandon, R.A., 1965, A new race of the neotenic salamander Gyrinophilus palleucus: Copeia, v. 1965, p. 346-352.

Brandon, R.A., 1966, Systematics of the Salamander Genus Gyrinophilus: Urbana, University of Illinois Press, Illinois Biological Monographs $35,82 \mathrm{p}$.

Buhlmann, K.A., 2001, A biological inventory of eight caves in northwestern Georgia with conservation implications: Journal of Cave and Karst Studies, v. 63, p. 91-98.

Caldwell, R.S., and Copeland, J.E., 1992, Status and habitat of the Tennessee cave salamander, Gyrinophilus palleucus: Unpublished report to Tennessee Wildlife Resources Agency, $24 \mathrm{p}$.

Camp, C.D., and Jensen, J.B., 2007, Use of twilight zones of caves by plethodontid salamanders: Copeia, v. 2007, p. 594-604. doi:10.1643/ 0045-8511(2007)2007[594:UOTZOC]2.0.CO;2.

Chirstman, M.C., and Culver, D.C., 2001, The relationship between cave biodiversity and available habitat: Journal of Biogeography, v. 28, p. 367-380. doi:10.1046/j.1365-2699.2001.00549.x.

Cliburn, J.W., and Middleton, A.L., Jr., 1983, The vertebrate cave fauna of Mississippi caves: NSS Bulletin, v. 45, p. 45-48.

Coles, V., 1944, Nesting of the turkey vulture in Ohio caves: The Auk, v. 61, p. $219-228$.

Conoway, C.H., and Howell, J.C., 1953, Observations on the mammals of Johnson and Carter counties, Tennessee, and Avery County in North Carolina: Journal of the Tennessee Academy of Science, v. 28, p. 53-61.

Cope, E.D., and Packard, A.S., Jr., 1881, The fauna of the Nickajack Cave: The American Naturalist, v. 15, p. 877-882.

Culver, D.C., Pipan T., 2009, The Biology of Caves and Other Subterranean Habitats: Oxford University Press, 254 p.

Culver, D.C., Christman, M.C., Elliott, W.R., Hobbs III, H.H., and Reddell, J.R., 2003, The North American obligate cave fauna: Regional patterns: Biodiversity \& Conservation, v. 12, p. 441-468. doi:10.1023/A:1022425908017.

Culver, D.C., Deharveng, L., Bedos, A., Lewis, J.J., Madden, M., Reddell, J.R., Sket, B., Trontelj, P., and White, D., 2006, The mid-latitude biodiversity ridge in terrestrial cave fauna: Ecography, v. 29, p. 120128. doi:10.1111/j.2005.0906-7590.04435.x.

Dearolf, K., 1956, Survey of North American cave vertebrates: Proceedings of the Pennsylvania Academy of Science, v. 30, p. 201-210. 
Dixon, G.B., and Zigler, K.S., 2011, Cave-obligate biodiversity on the campus of Sewanee: The University of the South, Franklin County, Tennessee: Southeastern Naturalist, v. 10, p. 251-266. doi:10.1656/ 058.010.0206.

Dodd, C.K. Jr., Griffey, M.L., and Corser, J.D., 2001, The cave associated amphibians of Great Smoky Mountains National Park: Review and monitoring: Journal of the Elisha Mitchell Scientific Society, v. 117, p. $139-149$.

Espinasa, L., and Jeffery, W.R., 2003, A troglomorphic sculpin (Pisces: Cottidae) population: Geography, morphology and conservation status: Journal of Cave and Karst Studies, v. 65, p. 93-100.

Espinasa, L., Mendyk, A., Schaffer, E., and Cahill, A., 2013, The second northernmost cave-adapted fish in the world? Groundwork on the Tytoona Cave sculpin population: Northeastern Naturalist, v. 20, p. $185-196$.

Etnier, D.A., and Starnes, W.C., 1993, The Fishes of Tennessee: Knoxville, Tennessee, University of Tennessee Press, 696 p.

Evermann, B.W., and Hildebrand, S.F., 1914, Notes on the fishes of east Tennessee: Bulletin of the United States Bureau of Fisheries, v. 34, p. 433-451.

Faber-Langendoen, D., Nichols, J., Master, L., Snow, K., Tomaino, A., Bittman, R., Hammerson, G., Heidel, B., Ramsay, L., Teucher, A., and Young, B., 2012, NatureServe Conservation Status Assessments: Methodology for Assigning Ranks, revised edition: Arlington, Virginia, NatureServe, $44 \mathrm{p}$.

Flock, B., 2013, 2013 Tennessee Bat Population Monitoring and White Nose Syndrome: Nashville, Tennessee Wildlife Resources Agency, TWRA Wildlife Technical Report 13-22, 17 p.

Flock, B., 2014, 2014 Bat Population Monitoring and White Nose Syndrome Surveillance: Nashville, Tennessee Wildlife Resources Agency, TWRA Wildlife Technical Report 14-07, 15 p.

Franz, R., Bauer, J., and Morris, T., 1994, Review of biologically significant caves and their faunas in Florida and south Georgia: Brimleyana, v. 20, p. 1-109.

Frick, W.F., Pollock, J.F., Hicks, A.C., Langwig, K.E., Reynolds, D.S., Turner, G.G., Butchkoski, C.M., and Kunz, T.H., 2010, An emerging disease causes regional population collapse of a common North American bat species: Science, v. 329, p. 679-682. doi:10.1126/ science. 1188594.

Garton, E.R., Grady, F., and Carey, S.D., 1993, The Vertebrate Fauna of West Virginia Caves: West Virginia Speleological Survey, Bulletin 11, $107 \mathrm{p}$.

Godwin, J.C., 2008, Biological Inventory of the Cave and Karst Systems of The Nature Conservancy's Sharp-Bingham Mountain Preserve: Auburn, Alabama, Alabama Natural Heritage Program, 40 p.

Gorički, Š., Niemiller, M.L., and Fenolio, D.B., 2012, Salamanders, in White, W.B. and Culver, D.C. eds., Encyclopedia of Caves, 2nd Edition, Waltham, Massachusetts, Elsevier, p. 665-676.

Harvey, M.J., 1994, Status of summer colonies of the endangered gray bat, Myotis grisescens, in Tennessee: Unpublished report, Tennessee Wildlife Resources Agency, $44 \mathrm{p}$.

Harvey, M.J., and Britzke, E.R., 2002, Distribution and status of endangered bats in Tennessee: Unpublished report, Tennessee Wildlife Resources Agency, $43 \mathrm{p}$.

Hay, W.P., 1902, Observations on the crustacean fauna of Nickajack Cave, Tennessee, and vicinity: Proceedings of the United States National Museum, v. 25, p. 417-439.

Himes, J., Beckett, D.C., and Trousdale, A.W., 2004, Survey of salamanders in Mississippi limestone caves: Southeastern Naturalist, v. 3, p. 241-248. doi:10.1656/1528-7092(2004)003[0241:SOSIML]2.0.CO;2.

Holliday, C., 2012, 2012 White-Nose Syndrome Disease Surveillance and Bat Population Monitoring Report. A Report of the Tennessee WNS Response Cooperators: Tennessee Chapter of The Nature Conservancy, $10 \mathrm{p}$.

Holsinger, J.R., and Culver, D.C., 1988, The invertebrate cave fauna of Virginia and a part of eastern Tennessee: zoogeography and ecology: Brimleyana, no. 14, p. 1-162.

Ingersoll, T.E., Sewall, B.J., and Amelon, S.K., 2013, Improved analysis of long-term monitoring data demonstrates marked regional declines of bat populations in the eastern United States: PLOS One, v. 8, art. e65907, 12 p. doi:10.1371/journal.pone.0065907.

International Union for the Conservation of Nature, 2012, IUCN Red List Categories and Criteria: Version 3.1. Second edition: Gland, Switzerland, and Cambridge, UK, IUCN, 32 p.
Ives, J.D., 1951, Faunal abundance cycle in a small crepuscular cave: Journal of the Elisha Mitchell Scientific Society, v. 67, p. 259-260.

Jones, R.S., and Hettler, W.F., 1959, Bat feeding by Green Sunfish: Texas Journal of Science, v. 11, p. 48.

Kennedy, M.L., Kennedy, P.K., Warr, E.L., and Wyatt, R.L., 2012, Annotated checklist of the mammals of Tennessee: Journal of the Tennessee Academy of Science, v. 87, p. 90-99.

Kuehne, R.A., 1966, Depauperate fish faunas of sinking creeks near Mammoth Cave, Kentucky: Copeia, v. 1966, p. 306-311.

Lamb, J., and Wyckoff, G., 2010, White-Nose Syndrome monitoring and response report for Tennessee, $27 \mathrm{p}$.

Lewis, J.J., 2005, Bioinventory of Caves of the Cumberland Escarpment Area of Tennessee. Final Report to Tennessee Wildlife Resources Agency \& The Nature Conservancy of Tennessee: Lewis \& Associates, $158 \mathrm{p}$.

Lewis, J.J., 2004, Bioinventory of Caves of the North Cumberland Project Area of Tennessee: Tennessee Chapter of the Nature Conservancy, $63 \mathrm{p}$.

Lewis, J.J., 1998, The Subterranean Fauna of the Blue River Area: Species at Risk Program, United States Geological Survey, The Nature Conservancy, and Natural Heritage Program, Indiana DNR, Division of Nature Preserves, $240 \mathrm{p}$.

Lewis, J.J., Whitaker, J.O., Jr., and Krantz, G.W., 2010, A biological reconnaissance of the invertebrate fauna of twelve Tennessee caves with notes on the guanophilic mites of the genus Macrocheles: Journal of the Tennessee Academy of Science, v. 85, p. 53-61.

Lyle, R.B., 1931, Black vulture nesting in Washington Co., Tenn.: The Auk, v. 48, p. 598.

Maher, S.P., Kramer, A.M., Pulliam, J.T., Zokan, M.A., Bowden, S.E., Barton, H.D., Magori, K., and Drake, J.M., 2012, Spread of whitenose syndrome on a network regulated by geography and climate: Nature Communications, v. 3, art. 1306. doi:10.1038/ncomms2301.

Martin, C.O., 2007, Assessment of the Population Status of the Gray Bat (Myotis grisescens). Status review, DoD Initiatives, and the Results of a Multi-Agency Effort to Survey Winter Populations at Major Hibernacula, 2005-2007: U.S. Army Corps of Engineers, Engineer Research and Development Center, Environmental Laboratory Final Report ERDC/EL TR-07-22, 97 p.

Mays, J.D., 2002, A systematic approach to sampling the arthropod assemblage of Gregory's Cave, Great Smoky Mountains National Park [Master's thesis]: Western Carolina University, 83 p.

McDaniel, V.R., and Gardner, J.E., 1977, Cave fauna of Arkansas: vertebrate taxa: Proceedings of the Arkansas Academy of Science, v. 31 , p. $68-71$.

Miller, B.T., Miller, J.L., Campbell, D.L., and Wyatt, P., 1998, Plethodon dorsalis. Reproduction: Herpetological Review, v. 29, p. 38-39.

Miller, B.T., and Niemiller, M.L., 2007, Distribution and Phylogenetics of the Tennessee Cave Salamander Complex (Gyrinophilus p. palleucus, G. p. necturoides, and G. gulolineatus): Nashville, Tennessee Wildlife Resources Agency unpublished technical report, $45 \mathrm{p}$.

Miller, B.T., and Niemiller, M.L., 2008, Distribution and relative abundance of Tennessee Cave Salamanders (Gyrinophilus palleucus and Gyrinophilus gulolineatus) with an emphasis on Tennessee populations: Herpetological Conservation and Biology, v. 3, p. 1-20.

Miller, B.T., and Niemiller, M.L., 2005, Pseudotriton ruber. Reproduction: Herpetological Review, v. 36, p. 429.

Miller, B.T., Niemiller, M.L., and Reynolds, R.G., 2008, Observations on egg-laying behavior and interactions among attending female Red Salamanders (Pseudotriton ruber) with comments on the use of caves by this species: Herpetological Conservation and Biology, v. 3, p. $203-210$.

Minnis, A.M., and Lindner, D.L., 2013, Phylogenetic evaluation of Geomyces and allies reveals no close relatives of Pseudogymnoascus destructans, comb. nov., in bat hibernacula of eastern North America: Fungal Biology, v. 117, p. 638-649. doi:10.1016/j.funbio.2013.07.001.

Mohr, C.E., 1952, A cave record for the red bat, Lasiurus b. borealis: Bulletin of the National Speleological Society, v. 14, p. 62-63.

Myers, R.F., 1960, Lasiurus from Missouri caves: Journal of Mammalogy, v. 41, p. 114-117. doi:10.2307/1376526.

Niemiller, M.L., Fenolio, D., Graening, G.O., and Miller, B.T., 2009a, Observations on oviposition and reproduction of the cave salamander, Eurycea lucifuga (Caudata: Plethodontidae), from Arkansas and Tennessee, USA: Speleobiology Notes, v. 1, p. 17-19. doi:10.5563/ spbn.vli1.9. 
Niemiller, M.L., Fitzpatrick, B.M., and Miller, B.T., 2008, Recent divergence with gene flow in Tennessee cave salamanders (Plethodontidae: Gyrinophilus) inferred from gene genealogies: Molecular Ecology, v. 17, p. 2258-2275. doi:10.1111/j.1365-294X.2008.03750.x.

Niemiller, M.L., Glorioso, B.M., Gray, E., Miller, B.T., Jensen, J.B., and Keyes, T., 2006, Pseudotriton ruber ruber. Size and subterranean aggregation: Herpetological Review, v. 37, p. 438.

Niemiller, M.L., Glorioso, B.M., and Miller, B.T., 2006, An eastern and subterranean maximum size record of the banded sculpin, Cottus carolinae, from Tennessee: Journal of the Tennessee Academy of Science, v. 81, p. 41-43.

Niemiller, M.L., and Miller, B.T., 2009, A survey of the cave-associated herpetofauna of the eastern United States with an emphasis on salamanders, in Proceedings of the 15th International Congress of Speleology, Kerrville, Texas, p. 249-256.

Niemiller, M.L., and Miller, B.T., 2011, Berry Cave Salamander. Gyrinophlus gulolineatus, in Niemiller, M.L. and Reynolds, R.G. eds., The Amphibians of Tennessee, Knoxville, Tennessee, University of Tennessee Press, p. 172-174.

Niemiller, M.L., and Miller, B.T., 2010, Gyrinophilus gulolineatus Brandon: Berry Cave Salamander: Catalogue of American Amphibians and Reptiles, no. 862, 4 p.

Niemiller, M.L., and Miller, B.T., 2008, Plethodon dorsalis. Reproduction: Herpetological Review, v. 39, p. 335

Niemiller, M.L., and Miller, B.T., 2005, Rana clamitans melanota. Predation: Herpetological Review, v. 36, p. 440

Niemiller, M.L., and Miller, B.T., 2007, Subterranean reproduction of the Southern Two-lined Salamander (Eurycea cirrigera) from Short Mountain, Tennessee: Herpetological Conservation and Biology, v. 2 , p. $106-112$.

Niemiller, M.L., Miller, B.T., and Fitzpatrick, B.M., 2010, Review of the scientific literature and research for the USFWS review for potential listing of the Berry Cave salamander (Gyrinophilus gulolineatus): unpublished technical report U.S. Fish and Wildlife Service, $22 \mathrm{p}$.

Niemiller, M.L., Miller, B.T., and Fitzpatrick, B.M., 2009b, Systematics and evolutionary history of subterranean Gyrinophilus salamanders, in Proceedings of the 15th International Congress of Speleology, Kerrville, Texas, p. 242-248.

Niemiller, M.L., and Reeves, W.C., 2014, Predation by the stygophilic crayfish Cambarus tenebrosus on the salamander Pseudotriton ruber within a cave in Cannon County, Tennessee, USA: Speleobiology Notes, v. 6, p. 8-13. doi:10.5563/spbn.v6i0.62.

Niemiller, M.L., and Reynolds, R.G., eds, 2011, The Amphibians of Tennessee, Knoxville, Tennessee, University of Tennessee Press, $369 \mathrm{p}$.

Niemiller, M.L., Reynolds, R.G., and Miller, B.T., eds, 2013, The Reptiles of Tennessee: Knoxville, Tennessee, University of Tennessee Press, $347 \mathrm{p}$

Niemiller, M.L., and Zigler, K.S., 2013, Patterns of cave biodiversity and endemism in the Appalachians and Interior Plateau of Tennessee, USA: PLOS ONE, v. 8, art. e64177, 17 p. doi:10.1371/journal. pone. 0064177 .

Osbourn, M.S., 2005, The natural history, distribution, and phenotypic variation of cave-dwelling spring salamanders, Gyrinophilus spp. Cope (Plethodontidae), in West Virginia [Master's thesis]: Marshall University, $207 \mathrm{p}$.

Pasek, J., 2015, weights: Weighting and Weighted Statistics, R package version 0.80

Pearson, W.D., and Boston, C.H., 1995, Distribution and Status of the Northern Cavefish, Amblyopsis spelaea: Final Report to the Indiana Department of Natural Resources Division of Fish and Wildlife, Nongame and Endangered Wildlife Program: Louisville, Water Resources Laboratory, University of Louisville, 198 p.

Poly, W.J., 2001, Nontroglobitic fishes in Bruffey-Hills Creek Cave, West Virginia, and other caves worldwide: Environmental Biology of Fishes, v. 62, p. 73-83. doi:10.1023/A:1011889613271.

Poly, W.J., and Boucher, C.E., 1996, Nontroglobitic fishes in caves: Their abnormalities, ecological classification and importance: American Midland Naturalist, v. 136, p. 187-198. doi:10.2307/2426644.

Prather, J.W., and Briggler, J.T., 2001, Use of small caves by anurans during a drought period in the Arkansas Ozarks: Journal of Herpetology, v. 35, p. 675-678. doi:10.2307/1565910.
Quay, W.B., and Miller, J.S., 1955, Occurrence of the red bat, Lasiurus borealis, in caves: Journal of Mammalogy, v. 36, p. 454-455. doi: $10.2307 / 1375691$

Rabinowitz, A., 1981, Thermal preference of the eastern pipistrelle bat (Pipistrellus subflavus) during hibernation: Journal of the Tennessee Academy of Science, v. 56, p. 113-114.

Rabinowitz, A., and Nottingham, B., 1979, Human visitation and fallwinter usage by bats in the Great Smoky Mountains National Park: Unpublished report to the National Park Service, $31 \mathrm{p}$.

Ray, C.K., Tan, M., and Armbruster, J.W., 2014, First record of Chrosomus erythrogaster (Cypriniformes: Cyprinidae) in the Mobile Basin: Southeastern Naturalist, v. 13, p. N33-N36. doi:10.1656/ 058.013.0402.

R Core Team, 2014, The R Project for Statistical Computing: Vienna, Austria, R Foundation for Statistical Computing, http://www. R-project.org. [accessed June 29, 2015]

Reese, A.M., 1934, The fauna of West Virginia caves: Proceedings of the West Virginia Academy of Science, v. 7, p. 39-53.

Reeves, W.K., 2000, Invertebrate cavernicoles of the Great Smoky Mountains National Park, USA: Journal of the Elisha Mitchell Scientific Society, v. 116, n. 4, p. 334-343.

Relyea, K., and Sutton, B., 1973, Cave dwelling Yellow Bullheads in Florida: Florida Scientist, v. 36, p. 31-34.

Rheams, K.F., Moser, P.H., and McGregor, S.W., 1992, Geologic, hydrologic, and biologic investigations in Arrowwood, Bobcat, Matthews, and Shelta Caves and selected caves, Madison County, Alabama: Geological Survey of Alabama and U.S. Department of Interior Fish and Wildlife Service Cooperative Agreement No. 14-160004-90-967, $262 \mathrm{p}$.

Ruhl, M., 2005, Flow reversal events increase the abundance of nontroglomorphic fish in the subterranean rivers of Mammoth Cave National Park, Kentucky [Master's thesis]: Western Kentucky University, $33 \mathrm{p}$.

Samoray, S., 2011, 2011 white-nose syndrome monitoring and bat population survey of hibernacula in Tennessee: Tennessee Chapter of The Nature Conservancy, $29 \mathrm{p}$.

Schneider, K., and Culver, D.C., 2004, Estimating subterranean species richness using intensive sampling and rarefaction curves in a high density cave region in West Virginia: Journal of Cave and Karst Studies, v. 66, p. 39-45.

Simmons, D.D., 1976, A naturally metamorphosed Gyrinophilus palleucus (Amphibia, Urodela, Plethodontidae): Journal of Herpetology, v. 10, p. 255-257. doi:10.2307/1562990.

Simmons, D.D., 1975, The evolutionary ecology of Gyrinophilus palleucus [Master's thesis]: University of Florida, $106 \mathrm{p}$.

Sket, B., 2008, Can we agree on ecological classifications of subterranean animals? Journal of Natural History, v. 42, n. 21-22, p. 1549-1563. doi:10.1080/00222930801995762.

Taylor, N., and Mays, J., 2006, The salamanders Eurycea longicauda and Plethodon glutinosus in Gregorys Cave, TN: Monitoring and observations on ecology and natural history: Southeastern Naturalist, v. 5, p. 435-442. doi:10.1656/1528-7092(2006)5[435:TSELAP]2.0. $\mathrm{CO} ; 2$.

Turner, G.G., Reeder, D.M., and Coleman, J.T.H., 2011, A five-year assessment of mortality and geographic spread of white-nose syndrome in North American bats and a look to the future: Bat Research News, v. 52, p. 13-27.

United States Fish and Wildlife Service, 2013, Endangered and threatened wildlife and plants: 12-month finding on a petition to list the Eastern Small-footed Bat and the Northern Long-eared Bat as endangered or threatened species; listing the Northern Long-eared Bat as an endangered species;: Federal Register, v. 78, p. 61046-61080.

United States Fish and Wildlife Service, 2015, Endangered and threatened wildlife and plants; threatened species status for the Northern Long-eared Bat with 4(d) rule: Federal Register, v. 80, p. 17974-18033.

United States Fish and Wildlife Service, 2007, Indiana Bat (Myotis sodalis) draft recovery plan: first revision: United States Fish and Wildlife Service, $258 \mathrm{p}$

Verant, M.L., Boyles, J.G., Waldrep, W., Jr., Wibbelt, G., and Blehert, D.S., 2012, Temperature-dependent growth in Geomyces destructans, the fungus that causes bat White-Nose Syndrome: PLOS ONE, v. 7, art. e46280, 7p. doi:10.1371/journal.pone.0046280. 
Wakefield, K.R., and Zigler, K.S., 2012, Obligate subterranean fauna of Carter State Natural Area, Franklin County, Tennessee: Speleobiology Notes, v. 4, p. 24-28. doi:10.5563/spbn.v4i0.38.

Wallace, R.L., 1984, Biological survey report of the Great Smoky Mountains National Park caves: Unpublished report to Great Smoky Mountains National Park, 34 p.

Wallace, R.L., 2003, Biological survey report of the Great Smoky Mountains National Park caves: Tennessee Caver, v. 2, p. 3-23.

Wallace, R.L., 1989, Report on a carabid cave beetle survey: Unpublished report to U.S. Fish and Wildlife Service, 6 p.
Weary, D.J., and Doctor, D.H., 2014, Karst in the United States: A digital map collection and database: U.S. Geological Survey Open-File Report 2014-1156, 23 p. doi:10.3133/ofr20141156.

Withers, D.I., 2009, A Guide to the Rare Animals in Tennessee: Nashville, Tennessee Natural Heritage Program, Division of Natural Areas, and Tennessee Department of Environment and Conservation, $61 \mathrm{p}$.

Wyckoff, G.R., and Niemiller, M.L., 2011, Spotted Dusky Salamander, in Niemiller, M.L., and Reynolds, R.G, eds., The Amphibians of Tennessee: Knoxville, University of Tennessee Press, p. 113-115. 